PDES, SUBMANIFOLDS AND

AFFINE DIFFERENTIAL GEOMETRY

BANACH CENTER PUBLICATIONS, VOLUME 57

INSTITUTE OF MATHEMATICS

POLISH ACADEMY OF SCIENCES

WARSZAWA 2002

\title{
HOMOGENEOUS SURFACES IN THE EQUIAFFINE SPACE $\mathbf{R}^{4}$
}

\author{
ROLF WALTER \\ Fachbereich Mathematik, Universität Dortmund \\ D-44221 Dortmund, Germany \\ E-mail: rolf.walter@math.uni-dortmund.de
}

Dedicated to Professor Martin Barner on the occasion of his 80th birthday

1. Introduction. In this paper we study nondegenerate surfaces in $\mathbf{R}^{4}$ which are homogeneous in the sense that they are orbits of a two-dimensional Lie subgroup of the special affine group of $\mathbf{R}^{4}$. We shall determine all such homogeneous surfaces, resulting in normal forms for the corresponding infinitesimal generators and for the orbits. An effort is made to reduce as far as possible these normal forms w.r.t. similarity and congruence, in particular to minimize the number of parameters within each class. There will result 35 group classes with some additional subcases for the orbits.

So far, nondegenerate equiaffinely homogeneous surfaces have been determined under the additional assumption of flatness (Wang [9], [12]), based on differential geometric methods. The general case has not been known, and it was stated (e.g. in Liu [3]) that this is a difficult problem. The parabolic equiaffinely homogeneous surfaces were classified in Walter [6].

The method we use here is Lie group oriented. To a certain extent it relies on the corresponding classification for linearly homogeneous surfaces in $\mathbf{R}^{4}$ as given in Walter [5]. However there are additional difficulties to overcome, caused by the higher dimension of the affine group and by the fact that Jordan normal forms only apply to single linear endomorphisms. Notice that the simultaneous normal form problem for two or more vector space endomorphisms is generally unsolved (Kirillov [2], p. 91). A fortiori this is true for two or more affine maps.

2. Differential-geometric notions and design of generators. Let $F: M \rightarrow \mathbf{R}^{4}$ be a $C^{\infty}$-immersion of a two-dimensional manifold $M$ into $\mathbf{R}^{4}$, where $\mathbf{R}^{4}$ is equipped with

2000 Mathematics Subject Classification: 53A15, 53C30.

The paper is in final form and no version of it will be published elsewhere. 
the usual determinant, denoted by brackets, but without any scalar product. We study properties of $F$ which are invariant in this setting, i.e. under the special affine group of $\mathbf{R}^{4}$. So we pursue equiaffine differential geometry for surfaces in $\mathbf{R}^{4}$.

A starting point for this theory is a conformal class of metrics invented by BurstinMayer [1]. Using a local base field $\mathcal{U}=(U, V)$ on $M$, a representative of the conformal class is

$$
G_{U, V}(X, Y):=\frac{1}{2}\left(\left[d_{U} F, d_{V} F, d_{X}\left(d_{U} F\right), d_{Y}\left(d_{V} F\right)\right]+\left[d_{U} F, d_{V} F, d_{Y}\left(d_{U} F\right), d_{X}\left(d_{V} F\right)\right]\right),
$$

where $X, Y$ are argument vector fields on $M$. The symbol $d$ denotes the differential of $\mathbf{R}^{4}$-valued functions on $M$. This expression is bilinear and symmetric in $X, Y$ and conformally invariant under any change of the frame field $\mathcal{U}$. The immersion $F$ is called elliptic, resp. hyperbolic if $G_{\mathcal{U}}$ is definite, resp. indefinite. If $G_{\mathcal{U}}$ has rank 1 , then $F$ is called parabolic.

Here we assume the metric to be nondegenerate, i.e. elliptic or hyperbolic. Then one can use the Gramian $\delta(U, V):=G_{U, V}(U, U) G_{U, V}(V, V)-\left(G_{U, V}(U, V)\right)^{2}$ in order to rescale $G_{\mathcal{U}}$ by $g:=(\delta(U, V))^{-1 / 3} G_{U, V}$, thus obtaining a metric $g$ which is independent of the choice of $\mathcal{U}$, hence globally defined. This $g$ is the equiaffine or Burstin-Mayer metric. It associates to the immersion $F$ an inner geometry which comes from equiaffine data alone. This is a remarkable analogy to Euclidean submanifold theory. Here, $g$ may be definite or indefinite. So the notions of pseudo-Riemannian geometry have to be applied in general.

If $U, V$ are the coordinate vector fields of a chart $(u, v)$ of $M: U:=\partial / \partial u, V:=\partial / \partial v$, then the above quantities take the classical form:

$$
\begin{gathered}
G_{U, V}=G_{11} d u^{2}+2 G_{12} d u d v+G_{22} d v^{2} \\
G_{11}:=\left[F_{u}, F_{v}, F_{u u}, F_{u v}\right], \quad 2 G_{12}:=\left[F_{u}, F_{v}, F_{u u}, F_{v v}\right], \quad G_{22}:=\left[F_{u}, F_{v}, F_{u v}, F_{v v}\right],
\end{gathered}
$$

the indices with $F$ denoting ordinary partial derivatives of $\mathbf{R}^{4}$-valued functions on $M$. For simplicity we sometimes write $G$ instead of $G_{\mathcal{U}}$ and $\delta$ instead of $\delta_{\mathcal{U}}$ and call $G$ the premetric and $\delta$ its discriminant. The discriminant decides on the definiteness of $G$ or $g$.

There are several recent approaches to the equiaffine theory of nondegenerate surfaces in $\mathbf{R}^{4}$ (Nomizu-Vrancken [4], Wang [8], [9], [10]). A unifying theory under the aspects of gauge theory and isotropy will be developed in Walter [7]. For the present context the above metric feature is sufficient.

The Lie group basics are very much similar to those described in the vector space setting of Walter [5], but in addition we have to take regard of the affine situation. The Lie algebra of a Lie group $\Gamma$ will be denoted by $\Gamma^{\bullet}$.

The main groups here are the affine, resp. special affine groups $\mathbf{G A}:=\mathbf{G A}(n, \mathbf{R})$, resp. SA $:=\mathbf{S A}(n, \mathbf{R})$, consisting of all affine maps $\lambda: \mathbf{R}^{n} \rightarrow \mathbf{R}^{n}$ which are bijective, resp. of determinant $1(n=4$, here). All Lie groups to be considered are subgroups of GA and correspondingly all Lie algebras are Lie subalgebras of $\mathbf{G A}^{\bullet}$.

An affine map $\lambda$ in general has the form $\lambda(z)=L z+l$ where $L: \mathbf{R}^{n} \rightarrow \mathbf{R}^{n}$ is linear and $l$ fixed in $\mathbf{R}^{n}$. The linear part $L$ is the total derivative $\lambda^{\prime}$ of $\lambda ; l$ is called the translation part. We set $\operatorname{det} \lambda:=\operatorname{det} L$. The derivative commutes with the composition (chain rule) 
and hence with the bracket for maps: $\left[\lambda_{1}, \lambda_{2}\right]:=\lambda_{1} \circ \lambda_{2}-\lambda_{2} \circ \lambda_{1}$, i.e.

$$
\left[\lambda_{1}, \lambda_{2}\right]^{\prime}=\left[\lambda_{1}^{\prime}, \lambda_{2}^{\prime}\right]
$$

In particular, if $\Gamma^{\bullet}$ is a Lie algebra and we denote by $\Gamma^{\bullet \prime}$ its image under the derivative, then $\Gamma^{\bullet \prime}$ is again a Lie subalgebra, possibly of lower dimension.

The following is of general interest for handling affine situations within the linear framework of vector spaces: We represent points, resp. vectors of the space $\mathbf{R}^{n}$ by elements of $\mathbf{R}^{n+1}$ with last entry 1 , resp. 0 , both written as columns.

$$
\text { point of } \mathbf{R}^{n}:\left(\begin{array}{c}
z_{1} \\
\vdots \\
z_{n} \\
\hline 1
\end{array}\right), \quad \text { vector of } \mathbf{R}^{n}:\left(\begin{array}{c}
z_{1} \\
\vdots \\
z_{n} \\
\hline 0
\end{array}\right) \text {. }
$$

For an affine map $\lambda$ we view the linear part $L$ as $(n \times n)$-matrix and then represent $\lambda$ by an $(n+1) \times(n+1)$-matrix:

$$
\mathbf{L}:=\left(\begin{array}{ccc||c} 
& & & l_{1} \\
& L & & \vdots \\
& & & l_{n} \\
\hline 0 & \ldots & 0 & 1
\end{array}\right)=\left(\begin{array}{c||c}
L & l \\
\hline 0 & 1
\end{array}\right),
$$

where we also gave a suitable short-hand version. In general we use the notations $\mathbf{L}, L, l$ in the above arrangement of boldface, normal capital and small letters. We often write $\mathbf{L}$ instead of $\lambda$.

By this imbedding, affine maps in $\mathbf{R}^{n}$ can be treated as linear maps of $\mathbf{R}^{n+1}$. This is in particular true for the composition and for the Lie group objects. For example the affine group of $\mathbf{R}^{n}$ becomes the subgroup of $\mathbf{G L}(n+1, \mathbf{R})$ consisting of the matrices $\left(\begin{array}{l||l}L & l \\ \hline 0 & 1\end{array}\right)$ with nonsingular upper-left corner $L$, and its Lie algebra will consist of the matrices $\left(\begin{array}{c||c}L & l \\ \hline 0 & 0\end{array}\right)$ with arbitrary upper-left corner $L$. Also the determinants and traces carry over appropriately, and the same is true for the exponential map. The special affine group SA now obtains as its Lie algebra the last mentioned matrices with trace 0 of the upper-left corner, and the Lie algebra $\Gamma^{\bullet}$ of a Lie subgroup $\Gamma$ of $\mathbf{S A}$ will consist of a linear subspace of these matrices.

Observe that the affine mappings of $\mathbf{R}^{n}$ now are sitting in the affine subspace of the set of $(n+1)$-square matrices with last row $0, . ., 0,1$, while the elements of the corresponding vector subspace have a 0 there instead of the 1 .

Still shorter, we mostly write $\left(\begin{array}{c||c}L & l \\ \hline 0 & \eta\end{array}\right)=:(L \| l)_{\eta}$ for $\eta \in\{0,1\}$.

Considering just Lie subgroups is not enough for the question of orbits, because a fixed subgroup of SA may have orbits which are not affinely congruent; see e.g. Walter [5], Sect. 8. So w.r.t. the normal forms we are looking for, there will be two equivalence relations, one for the groups and one for the orbits: 
If $\mathbf{T} \in \mathbf{G A}$ is fixed and $\Gamma$ is a Lie subgroup of $\mathbf{S A}$ with Lie algebra $\Gamma^{\bullet}$ then $\mathbf{T} \Gamma^{-1}$ is a Lie subgroup of $\mathbf{S A}$ with Lie algebra $\mathbf{T} \Gamma^{\bullet} \mathbf{T}^{-1}$, called similar to $\Gamma^{\bullet}$. This similarity will play the role of equivalence for the Lie subgroups of $\mathbf{S A}$. Observe that any $\mathbf{T} \in \mathbf{G A}$ corresponds to a change of an affine frame, where the linear part $T$ describes the base change and the translation part the change of origin. For short, we call $\mathbf{T}$ a similarity. In particular we define the group of self-similarities of $\Gamma$ by

$$
\mathcal{F}_{\Gamma}:=\left\{\mathbf{T} \in \mathbf{G A} \mid \mathbf{T} \Gamma \mathbf{T}^{-1}=\Gamma\right\}
$$

Via the exponential map this is also expressible on the Lie algebra level:

$$
\mathcal{F}_{\Gamma}:=\left\{\mathbf{T} \in \mathbf{G A} \mid \mathbf{T} \Gamma^{\bullet} \mathbf{T}^{-1}=\Gamma^{\bullet}\right\} .
$$

An orbit of $\Gamma$ is of the form $\Gamma x_{0}$ for fixed $x_{0} \in \mathbf{R}^{n}$, the initial point. If we have two orbits $\Gamma x_{0}, \Gamma \widetilde{x}_{0}$ of the same group and if $\widetilde{x}_{0}=\mathbf{T} x_{0}$ for some $\mathbf{T} \in \mathcal{F}_{\Gamma}$ then $\Gamma \widetilde{x}_{0}=$ $\Gamma \mathbf{T} x_{0}=\mathbf{T} \Gamma x_{0}$. So the two orbits $\Gamma x_{0}$ and $\Gamma \widetilde{x}_{0}$ are congruent via $\mathbf{T}$. If moreover $\mathbf{T}$ has determinant 1 then they are congruent modulo SA. In order to reduce the number of representatives, we shall finally do the equivalence for orbits neglecting orientation, i.e. we use for this the group $\widetilde{\mathbf{S A}}$ of the $\mathbf{T} \in \mathbf{G A}$ with $\operatorname{det} \mathbf{T} \in\{1,-1\}$. Summing up, two initial points $x_{0}, \widetilde{x}_{0}$ are considered equivalent if there is a $\mathbf{T} \in \mathcal{F}_{\Gamma}$ with $|\operatorname{det} \mathbf{T}|=1$ such that $\widetilde{x}_{0}=\mathbf{T} x_{0}$. Then the orbits $\Gamma x_{0}, \Gamma \widetilde{x}_{0}$ are congruent modulo $\widetilde{\mathbf{S A}}$.

The normal form for a Lie algebra will consist of a basis of it whose entries are as simple as possible and expressing geometric properties if possible (e.g. invariance of subspaces, spectra, annihilation of translation parts, etc.). Any element in a Lie algebra basis will be called a generator and its one-dimensional span a ray.

In order to simplify a set of generators, we often use special types of similarities $\mathbf{T}=(T \| t)_{1}$ : the pure translational ones for which $T=I$ (= unit matrix), the pure linear ones for which $t=0$, and also the pure homothetic ones for which $t=0$ and $T=r I, r \neq 0$.

Sometimes it is useful to change just the translation part of an $\mathbf{L}=(L \| l)_{0}$ by a general homothety $\mathbf{T}=(r I \| t)$. Consider for this the equation

$$
\left(\begin{array}{c||c}
r I & t \\
\hline 0 & 1
\end{array}\right)\left(\begin{array}{c||c}
L & l \\
\hline 0 & 0
\end{array}\right)\left(\begin{array}{c||c}
r I & t \\
\hline 0 & 1
\end{array}\right)^{-1}=\left(\begin{array}{c||c}
L & r l-L t \\
\hline 0 & 0
\end{array}\right) .
$$

So, if $r=1$, the new translation part is $l_{n}=l-L t$, hence all $l_{n}$ in the affine subspace $l+\operatorname{im} L$ are possible. For example, we can achieve $l_{n}=0$ if $L$ is surjective. These possibilities for $l_{n}$ can also be applied blockwise. We call this a translation reduction. If $t=0$ then the new translation part is the $r$-multiple of the old one. We call this a homothety reduction.

Occasionally we meet the following situation: If $T$ is a matrix, transforming the linear part $L$ of $\mathbf{L}=(L \| l)_{0}$ to $L_{0}, T L T^{-1}=L_{0}$, then, with the corresponding pure linear $\mathbf{T}$, the $\mathbf{L}$ is transformed to $\left(L_{0} \| l_{0}\right)_{0}$ with $l_{0}:=T l$. We say for this that the translation part of $\mathbf{L}$ is taken along with such a $\mathbf{T}$.

In many instances, pure linear similarities, transforming the linear parts of given generators $\mathbf{E}, \mathbf{H}$ to normal forms, can be read off from Walter [5]. We adopt from there the nomenclature for Jordan normal forms of a single vector space endomorphism. In par- 
ticular, the numbering of Jordan block types follows the partition pattern of elementary Jordan blocks: A Jordan block is of type $\mathcal{J}_{i}(n)$ if its total size is $n$ and its elementary Jordan blocks correspond to the $i$-th partition in the sequence of lexicographically ordered partitions of $n$. The symbols $e_{1}, \ldots, e_{n}$ denote the standard base vectors of $\mathbf{R}^{n}$.

In [5] we also developed several techniques to handle special pairs of endomorphisms, in particular those with a nilpotent component (cf. Lemmas 3.2, 3.3, 4.1, 5.1, 5.2 there). For the present affine situation this may be supplemented by the following

2.1. LemmA. If the linear part of $\mathbf{L}=(L \| l)_{0}$ is a nilpotent Jordan block $L=J(n, 0)$ of maximal degree, then we can reach by pure translation reduction that the translation part becomes

$$
t(a):=(a, 0, \ldots, 0)^{\top} \in \mathbf{R}^{n} .
$$

If, in addition, $a \neq 0$, we can reach by a pure linear similarity of the form

$$
\mathbf{T}:=\operatorname{diag}\left(r^{m}, \ldots, r^{m-n+1}, 1\right), \quad m \in\{n-1, n\}
$$

applied to the r-multiple of $\mathbf{L}$ that $a=1$ :

$$
\mathbf{T r}(L \| t(a))_{0} \mathbf{T}^{-1}=(L \| t(1))_{0} .
$$

Proof. The first part is clear from the above because the image of $L$ is spanned by $e_{2}, \ldots, e_{n}$. For the second part, a matrix calculation yields

$$
\mathbf{T} r(L \| t(a))_{0} \mathbf{T}^{-1}=\left(L \| t\left(r^{m+1} a\right)\right)_{0} .
$$

Take $m=n$ if $n$ is even and $m=n-1$ if $n$ is odd to render $r^{m+1} a=1$ by choice of $r$.

Peculiarly important is the blockwise variant of this procedure. We call this a nilpotence reduction. (For a maximal nilpotent $L$ itself, the $\mathbf{T}$ in 2.1 could be replaced by a pure homothety.)

Usually, for our two-dimensional Lie algebras, linearly independent generators are denoted by $\mathbf{E}, \mathbf{H}$ and their linear parts by $E, H$. The span $\mathbf{E}, \mathbf{H}$ is also called a pencil, and that of $E, H$ the corresponding linear pencil. In view of (2.1), the latter is a base free construction from the former. If $\operatorname{span}(E, H)$ has dimension lower than 2 , there is a generator in $\operatorname{span}(\mathbf{E}, \mathbf{H})$ with vanishing linear part, and it is easy to see that the corresponding orbit lines are straight, which is impossible for a nondegenerate Burstin-Mayer metric. So in our context, the linear pencils are really two-dimensional. Two pencils, generated by $\mathbf{E}, \mathbf{H}$, resp. $\mathbf{E}^{\prime}, \mathbf{H}^{\prime}$, are similar iff there is a $\mathbf{T} \in \mathbf{G} \mathbf{A}$ and real $\alpha_{1}, \alpha_{2}, \beta_{1}, \beta_{2}$ with

$$
\begin{aligned}
\mathbf{T E}^{\prime} \mathbf{T}^{-1} & =\alpha_{1} \mathbf{E}+\alpha_{2} \mathbf{H} \\
\mathbf{T H}^{\prime} \mathbf{T}^{-1} & =\beta_{1} \mathbf{E}+\beta_{2} \mathbf{H}
\end{aligned}, \quad\left|\begin{array}{cc}
\alpha_{1} & \alpha_{2} \\
\beta_{1} & \beta_{2}
\end{array}\right| \neq 0 .
$$

We call this the similarity test. In particular, the group $\mathcal{F}_{\Gamma}$ of self-similarities for a pencil $\operatorname{span}(\mathbf{E}, \mathbf{H})$ is given by the solutions $\mathbf{T} \in \mathbf{G A}$ of

$$
\begin{aligned}
\mathbf{T E T}^{-1} & =\alpha_{1} \mathbf{E}+\alpha_{2} \mathbf{H} \\
\mathbf{T H T}^{-1} & =\beta_{1} \mathbf{E}+\beta_{2} \mathbf{H}
\end{aligned}, \quad\left|\begin{array}{cc}
\alpha_{1} & \alpha_{2} \\
\beta_{1} & \beta_{2}
\end{array}\right| \neq 0 .
$$

The classification of abstract two-dimensional Lie algebras is well known and easy: There are only two different types: either there are two generators $\mathbf{E}, \mathbf{H}$ with $[\mathbf{E}, \mathbf{H}]=0$ (commutative case) or with $[\mathbf{E}, \mathbf{H}]=\mathbf{E}$ (noncommutative case). 
Our general plan to gain all normal forms for two-dimensional Lie subalgebras of $\mathbf{G A} \mathbf{A}^{\bullet}$ is the following: We first look for all possible Jordan normal forms for the linear part of one of them, say $E$, try to simplify the translation part of $\mathbf{E}$ by translation reduction, then search all $H$ satisfying the commutator relations for $E, H([E, H]=0$, resp. $[E, H]=E$, and again try to simplify further both $\mathbf{E}, \mathbf{H}$ by suitable similarities. This latter is the really inventive part. Of course, always the commutator relations $[\mathbf{E}, \mathbf{H}]=0$, resp. $[\mathbf{E}, \mathbf{H}]=\mathbf{E}$ have to be taken into account. Moreover, during this procedure, the generators $\mathbf{E}, \mathbf{H}$ may be replaced by suitable linear combinations because only their span is important for the generated group.

An application of translation reductions, homothety reductions, nilpotence reductions, commutator relations or linear combinations of generators (possibly together) will be called a design, with the corresponding attribute: T, H, N, C, L. For example, a TCdesign is a combination of a translation reduction and an application of a commutator relation.

In the following concrete calculations the components of the translation part of $\mathbf{E}$, resp. $\mathbf{H}$ will be denoted by $E_{1}, \ldots, E_{4}$, resp. $H_{1}, \ldots, H_{4}$, in general.

3. The commutative nondiagonalizable case. Here we study two-dimensional commutative Lie subalgebras $\Gamma^{\bullet}$ of $\mathbf{G A}^{\bullet}$ (in dimension 4) whose orbits are not all degenerate and whose linear pencils $\Gamma^{\bullet \prime}$ contain at least one element which is not $\mathbf{C}$ diagonalizable. A useful addition to the T-design is the following:

3.1. Lemma. If a generator $\mathbf{E}$ has a nonsingular linear part $E$ then its translation part can be made zero by a pure translation similarity and then, in the commutative case $[\mathbf{E}, \mathbf{H}]=0$, the translation part of $\mathbf{H}$ is also necessarily zero.

Proof. The first part is clear from the introduction. Now, if $\mathbf{E}=(E \| 0)_{0}$ and $\mathbf{H}=$ $(H \| l)_{0}$, one calculates $0=[\mathbf{E}, \mathbf{H}]=([E, H] \| E l)_{0}$ hence $E l=0$, so $l=0$.

A. Theorem. Let $\Gamma^{\bullet}$ be a two-dimensional commutative Lie subalgebra of $\mathbf{S A}(4, \mathbf{R})^{\bullet}$, whose orbits are not all degenerate with respect to the Burstin-Mayer metric. Assume that not all elements of the corresponding linear pencil $\Gamma^{\bullet \prime}$ are $\mathbf{C}$-diagonalizable.

Then $\Gamma^{\bullet}$ is similar to one of the following twelve cases $\|1\|, \ldots,\|12\|$, specifying $\Gamma^{\bullet}$ by the given generators $\mathbf{E}, \mathbf{H}$. The nondegenerate orbits are specified by the given initial points $x_{0} \in \mathbf{R}^{4}$. Additionally, the discriminants $\delta$ at an arbitrary initial point and implicit equations of the orbits for $x_{0}$ are displayed.

Within each case, the parameters possibly occurring in $\mathbf{E}, \mathbf{H}$, resp. $x_{0}$ are separating under the given conditions between similarity classes of pencils, resp. congruence classes of orbits.

(I) $E$ is of type $\mathcal{J}_{1}(4)$ :

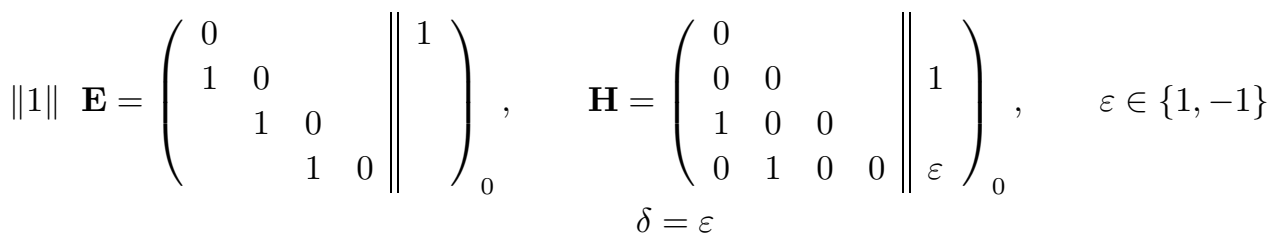




$$
x_{0}=(0,0,0,0)^{\top}, \quad\left\{\begin{aligned}
3 x_{3} & =3 x_{1} x_{2}-x_{1}^{3} \\
12 x_{4} & =6 x_{2}^{2}-x_{1}^{4}-6 \varepsilon\left(x_{1}^{2}-2 x_{2}\right) .
\end{aligned}\right.
$$

(II.1) $E$ is of type $\mathcal{J}_{2}(4)$ :

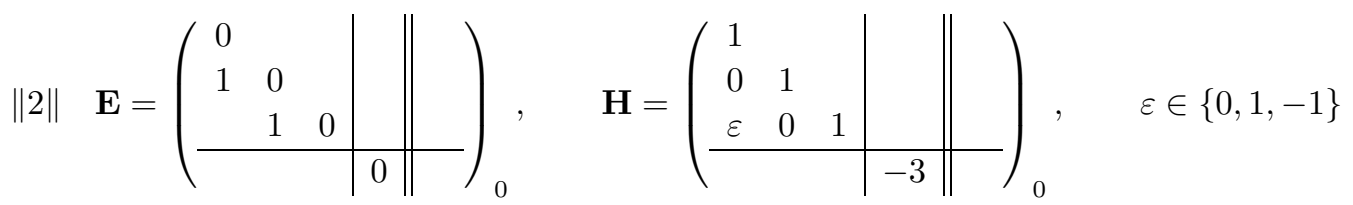

$$
\begin{aligned}
& \delta=-36 x_{1}^{6} x_{4}^{2} \\
& x_{0}=(1,0,0, c)^{\top}, \quad c>0, \quad\left\{\begin{array}{r}
x_{1}^{3} x_{4}=c \\
2 x_{1} x_{3}-x_{2}^{2}-2 \varepsilon x_{1}^{2} \ln x_{1}=0 .
\end{array}\right.
\end{aligned}
$$

(II.2) $E$ is of type $\mathcal{J}_{2}(4)$ :

$\|3\|$

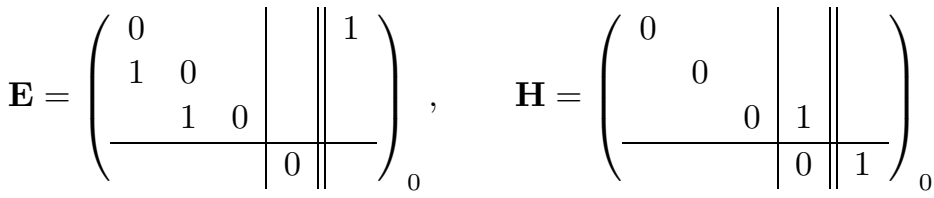

$$
\begin{aligned}
& \delta=-\frac{1}{4} \\
& x_{0}=(0,0,0,0)^{\top}, \quad\left\{\begin{array}{l}
2 x_{2}=x_{1}^{2} \\
6 x_{3}=x_{1}^{3}+3 x_{4}^{2} .
\end{array}\right.
\end{aligned}
$$

(III) $E$ is of type $\mathcal{J}_{3}(4)$ :

$\|4\|$

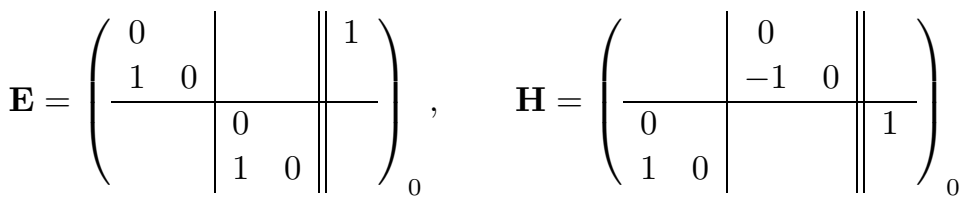

$$
\begin{aligned}
& \delta=1 \\
& x_{0}=(0,0,0,0)^{\top}, \quad\left\{\begin{aligned}
2 x_{2} & =x_{1}^{2}-x_{3}^{2} \\
x_{4} & =x_{1} x_{3} .
\end{aligned}\right.
\end{aligned}
$$

(IV.1) $E$ is of type $\mathcal{J}_{4}(4)$ :

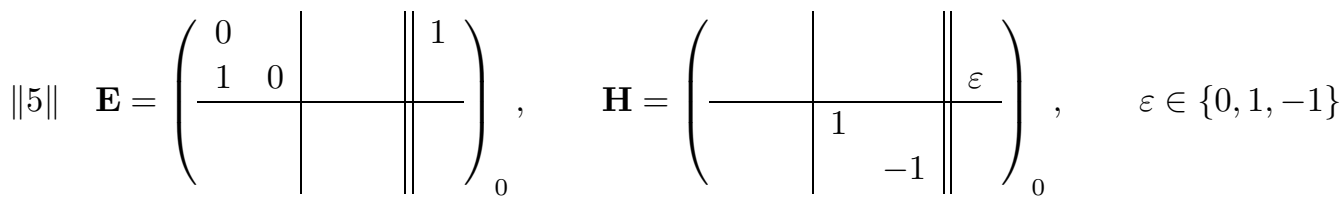

$$
\begin{aligned}
& \delta=-x_{3}^{2} x_{4}^{2} \\
& x_{0}=(0,0,1, c)^{\top}, \quad c>0, \quad\left\{\begin{aligned}
2 x_{2} & =x_{1}^{2}+2 \varepsilon \ln x_{3} \\
x_{3} x_{4} & =c .
\end{aligned}\right.
\end{aligned}
$$


(IV.2) $E$ is of type $\mathcal{J}_{4}(4)$ :

$\|6\|$

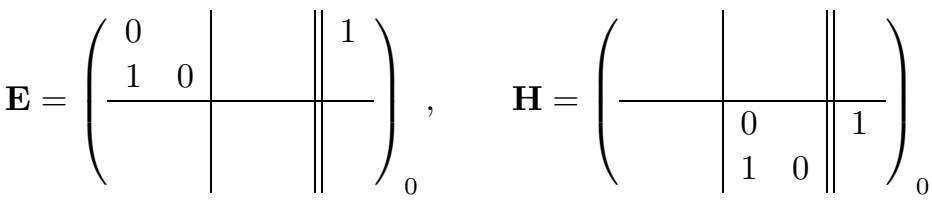

$$
\begin{aligned}
& \delta=-\frac{1}{4} \\
& x_{0}=(0,0,0,0)^{\top}, \quad\left\{\begin{array}{l}
2 x_{2}=x_{1}^{2} \\
2 x_{4}=x_{3}^{2} .
\end{array}\right.
\end{aligned}
$$

(V.1) $E$ is of type $\mathcal{J}_{1}(2)+\mathcal{J}_{1}(1)+\mathcal{J}_{1}^{\prime}(1)$ :

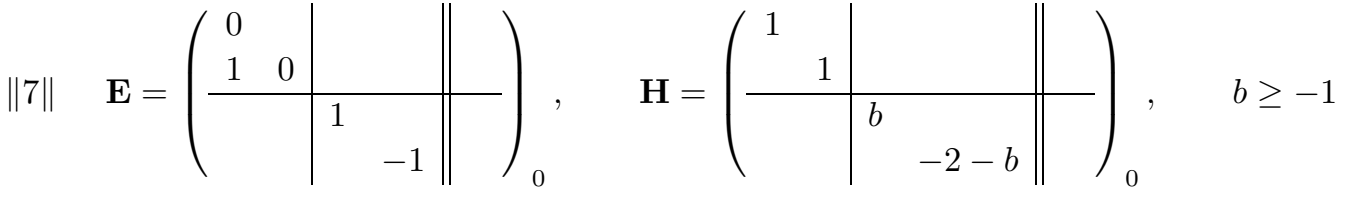

$$
\begin{aligned}
& \delta=-\left((b+1)^{2}+32\right) x_{1}^{4} x_{3}^{2} x_{4}^{2} \\
& x_{0}=(1,0,1, c)^{\top}, \quad c>0, \quad\left\{\begin{array}{r}
x_{1}^{2} x_{3} x_{4}=c \\
x_{2}+x_{1}\left(b \ln x_{1}-\ln x_{3}\right)=0 .
\end{array}\right.
\end{aligned}
$$

(V.2) $E$ is of type $\mathcal{J}_{1}(2)+\mathcal{J}_{1}(1)+\mathcal{J}_{1}^{\prime}(1)$ :

$\|8\|$

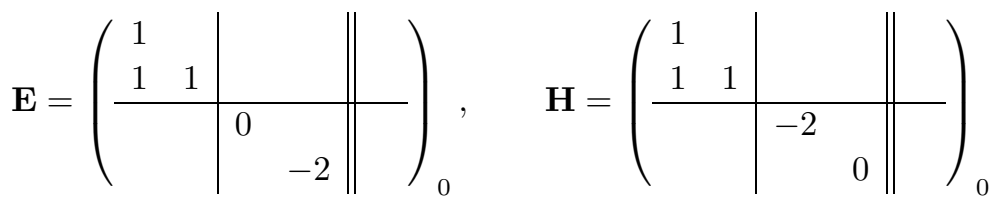

$$
\begin{aligned}
& \delta=-64 x_{1}^{4} x_{3}^{2} x_{4}^{2} \\
& x_{0}=(1,0,1, c)^{\top}, \quad c>0, \quad\left\{\begin{array}{r}
x_{1}^{2} x_{3} x_{4}=c \\
x_{2}-x_{1} \ln x_{1}=0 .
\end{array}\right.
\end{aligned}
$$

(VI) $E$ is of type $\mathcal{J}_{1}(2)+\mathcal{K}_{1}(2)$ :

$$
\begin{aligned}
& \|9\| \mathbf{E}=\left(\begin{array}{cc|c||c|c}
0 & & & \\
1 & 0 & & & \\
\hline & 0 & -1 \\
& 1 & 0
\end{array}\right)_{0}, \quad \mathbf{H}=\left(\begin{array}{cc|cc}
1 & & \\
& 1 & & \\
& & -1 & -b \\
& b & -1
\end{array}\right)_{0}, \quad b \geq 0, b \neq \sqrt{32} \\
& \delta=\frac{1}{4}\left(32-b^{2}\right) x_{1}^{4}\left(x_{3}^{2}+x_{4}^{2}\right)^{2} \\
& x_{0}=(c, 0,1,0)^{\top}, \quad c>0, \quad\left\{\begin{array}{r}
x_{1}^{2}\left(x_{3}^{2}+x_{4}^{2}\right)=c^{2} \\
\frac{x_{2}}{x_{1}}+b \ln \frac{x_{1}}{c}-\arctan \frac{x_{4}}{x_{3}}=0 .
\end{array}\right.
\end{aligned}
$$


(VII.1) $E$ is of type $\mathcal{J}_{2}(2)+\mathcal{K}_{1}(2)$ :

||10

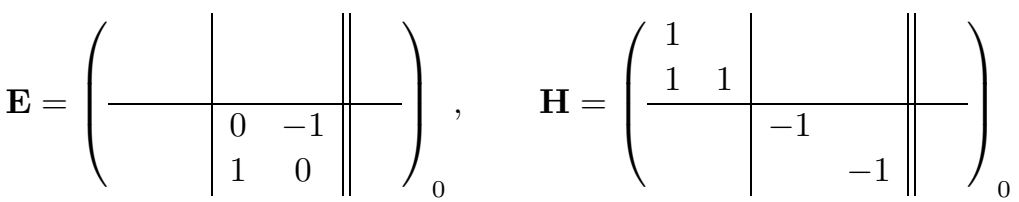

$$
\begin{aligned}
& \delta=-\frac{1}{4} x_{1}^{4}\left(x_{3}^{2}+x_{4}^{2}\right) \\
& x_{0}=(c, 0,1,0)^{\top}, \quad c>0, \quad\left\{\begin{array}{l}
x_{1}^{2}\left(x_{3}^{2}+x_{4}^{2}\right)^{2}=c^{2} \\
x_{2}-x_{1} \ln \frac{x_{1}}{c}=0 .
\end{array}\right.
\end{aligned}
$$

(VII.2) $E$ is of type $\mathcal{J}_{2}(2)+\mathcal{K}_{1}(2)$ :

||11\|

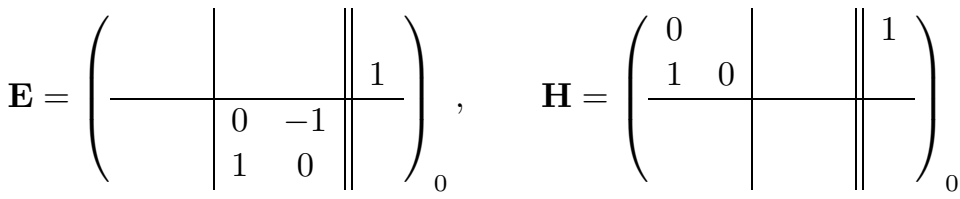

$$
\begin{aligned}
& \delta=-\frac{1}{4}\left(x_{3}^{2}+x_{4}^{2}\right)^{2} \\
& x_{0}=(0,0, c, 0)^{\top}, \quad c>0, \quad\left\{\begin{aligned}
x_{3}^{2}+x_{4}^{2} & =c^{2} \\
2 x_{2} & =x_{1}^{2}+2 \arctan \frac{x_{4}}{x_{3}} .
\end{aligned}\right.
\end{aligned}
$$

(VII.3) $E$ is of type $\mathcal{J}_{2}(2)+\mathcal{K}_{1}(2)$ :

$\|12\|$

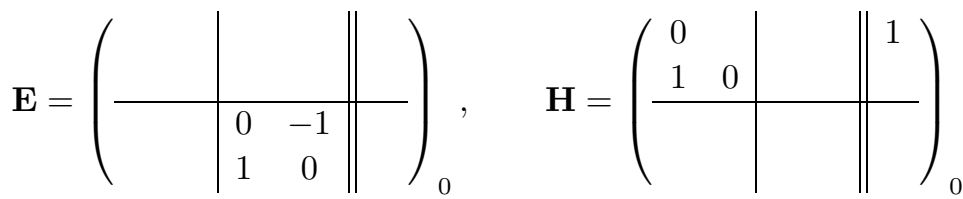

$$
\begin{aligned}
& \delta=-\frac{1}{4}\left(x_{3}^{2}+x_{4}^{2}\right)^{2} \\
& x_{0}=(0,0,1,0)^{\top}, \quad\left\{\begin{aligned}
x_{3}^{2}+x_{4}^{2} & =1 \\
2 x_{2} & =x_{1}^{2} .
\end{aligned}\right.
\end{aligned}
$$

REMARK. The specification of the generators $\mathbf{E}, \mathbf{H}$ and the initial point $x_{0}$ completely fixes the orbit because its parametrization is given by the $\mathbf{R}^{4}$-part of

$$
(u, v) \mapsto \exp (u \mathbf{E}) \exp (v \mathbf{H})\left(x_{0}, 1\right)^{\top}, \quad(u, v) \in \mathbf{R}^{2} .
$$

In all instances, this expression can be easily and explicitly computed from the given normal forms.

Proof. We carry out the procedure described above, considering those pencils $\operatorname{span}(\mathbf{E}, \mathbf{H})$ with commuting $\mathbf{E}, \mathbf{H}$ such that $\operatorname{span}(E, H)$ is of dimension 2 and its elements are not all $\mathbf{R}$-diagonalizable. We can put aside all cases with two linearly independent C-diagonalizable generators of $\operatorname{span}(E, H)$ because they are treated in the next section. We have to go through all possibilities of Jordan normal forms of non-R-diagonalizable endomorphisms for one of the generators, say $E$. The arguments will be given in full detail 
for many characteristic cases and indicated briefly in other cases which can be handled similarly or as in [5].

1) $E$ is of type $\mathcal{J}_{1}(4)$ : This case didn't occur in the vector space situation [5] because all orbits belonging to $\operatorname{span}(E, H)$ were contained in hyperplanes. However, the orbits belonging to $\operatorname{span}(\mathbf{E}, \mathbf{H})$ do not degenerate in general. Here we perform the reduction of $\operatorname{span}(\mathbf{E}, \mathbf{H})$ in a special way:

By the trace condition $E=J(4,0)$, and by T-design we can assume $E_{i}=0$ for $i=2, \ldots, 4$. By [5], Lemma 3.1, $H$ is known, and by subtracting from $\mathbf{H}$ a multiple of $\mathbf{E}$ we can assume

$$
\mathbf{E}=\left(\begin{array}{cccc||c}
0 & & & & E_{1} \\
1 & 0 & & & 0 \\
& 1 & 0 & & 0 \\
& & 1 & 0 & 0
\end{array}\right)_{0}, \quad \mathbf{H}=\left(\begin{array}{cccc||c}
0 & & & & H_{1} \\
0 & 0 & & & H_{2} \\
h_{2} & 0 & 0 & & H_{3} \\
h_{3} & h_{2} & 0 & 0 & H_{4}
\end{array}\right)_{0} .
$$

$[\mathbf{E}, \mathbf{H}]=0$ demands $H_{1}=0, H_{2}=h_{2} E_{1}, H_{3}=h_{3} E_{1}$, and the regularity of the premetric requires $E_{1} \neq 0, h_{2} \neq 0$, thus, by $\mathrm{N}$-design (Lemma 2.1), $E_{1}=1$ is possible. In summary, we can achieve, after renaming

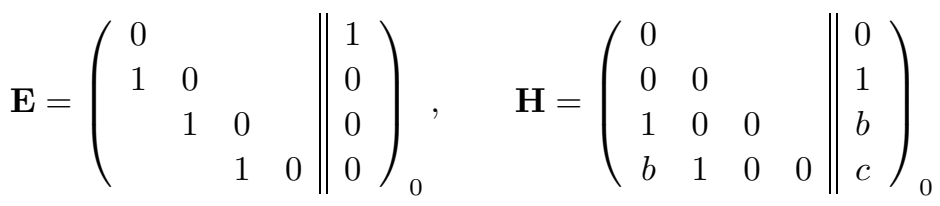

with $\delta=\frac{1}{4}\left(4 c-5 b^{2}\right)$, so $4 c-5 b^{2} \neq 0$. All elements in $\operatorname{span}(\mathbf{E}, \mathbf{H})$ are then nilpotent of degree 5, except for $\mathbf{H}$ which is nilpotent of degree 3, so distinguished by that.

Now we observe the following relation:

$$
\mathbf{H}=\mathbf{E}^{2}\left(\mathbf{I}+b \mathbf{E}+c \mathbf{E}^{2}\right),
$$

where $\mathbf{I}$ denotes the $(5 \times 5)$-unit matrix. This is the key for a further reduction of the parameters $b, c$ : With the polynomial

$$
P(\lambda):=\lambda^{2}\left(1+b \lambda+c \lambda^{2}\right),
$$

the relation may be written

$$
\mathbf{H}=P(\mathbf{E}) .
$$

By changing the base in the pencil via

$$
\begin{aligned}
\mathbf{E}^{\prime} & :=x \mathbf{E}+y \mathbf{H} \\
\mathbf{H}^{\prime} & :=z \mathbf{H}
\end{aligned} \quad x \neq 0, z \neq 0
$$

we try to get a simpler polynomial

$$
Q(\mu):=\mu^{2}\left(1+b^{\prime} \mu+c^{\prime} \mu^{2}\right)
$$

on the right hand side:

$$
\mathbf{H}^{\prime}=Q\left(\mathbf{E}^{\prime}\right) .
$$

Inserting here $\mathbf{E}^{\prime}, \mathbf{H}^{\prime}$ gives after some calculation, using $\mathbf{E}^{5}=0$ :

$$
\left(z c-2 x y b-3 x^{2} b^{\prime} y-x^{4} c^{\prime}-y^{2}\right) \mathbf{E}^{4}+\left(z b-x^{3} b^{\prime}-2 x y\right) \mathbf{E}^{3}+\left(z-x^{2}\right) \mathbf{E}^{2}=0 .
$$


This is equivalent to the vanishing of the coefficients of the occurring powers of $\mathbf{E}$, giving a system with the following recursive solution:

$$
z=x^{2}, \quad y=\frac{1}{2} x\left(b-b^{\prime} x\right), \quad\left(5 b^{\prime 2}-4 c^{\prime}\right) x^{2}=5 b^{2}-4 c .
$$

This is solvable for $x, y, z$ iff $\operatorname{sign}\left(4 c^{\prime}-5 b^{\prime 2}\right)=\operatorname{sign}\left(4 c-5 b^{2}\right)$. Thus if $4 c-5 b^{2}>0$ then $b^{\prime}=0, c^{\prime}=1$ is possible, and if $4 c-5 b^{2}<0$ then $b^{\prime}=0, c^{\prime}=-1$ is possible. If already $b, c$ have these values, then only $x= \pm 1, y=0, z=1$ are still possible. So we arrive at the normal forms as announced in $\|1\|$, and $\mathbf{E}, \mathbf{H}$ are unique up to a sign change of $\mathbf{E}$.

By this characterization, the self-similarities are those $\mathbf{T}$ for which there is a $\eta \in$ $\{1,-1\}$ with

$$
\mathbf{T E}=\eta \mathbf{E T}, \quad \mathbf{T H}=\mathbf{H T} .
$$

The solutions of these equations are calculated as

$$
\mathbf{T}=\left(\begin{array}{cccc||c}
\eta & & & \\
\eta \tau_{4} & 1 & & \\
\eta \tau_{3} & \tau_{4} & \eta & \\
\eta \tau_{2} & \tau_{3} & \eta \tau_{4} & 1 & \tau_{3} \\
\tau_{2} \\
\tau_{1}
\end{array}\right)_{1}
$$

with free parameters $\tau_{1}, \ldots, \tau_{4}$. It is easily seen that the $\mathbf{T}$ with $|\operatorname{det} \mathbf{T}|=1$ operate transitively on $\mathbf{R}^{4}$. So all orbits are congruent to each other, and we may pick one, say that with initial point $0 \in \mathbf{R}^{4}$. Its parametrization (in $\mathbf{R}^{5}$ ) is

$$
(u, v) \mapsto \exp (u \mathbf{H}) \exp (v \mathbf{E})\left(\begin{array}{c}
0 \\
0 \\
0 \\
0 \\
1
\end{array}\right)=\left(\begin{array}{c}
u \\
\frac{1}{2} u^{2}+v \\
\frac{1}{6} u^{3}+u v \\
\frac{1}{24} u^{4}+\frac{1}{2} u^{2} v+\frac{1}{2} v^{2}+\varepsilon v \\
1
\end{array}\right)
$$

By eliminating $u, v$, the implicit equations of this orbit (in $\mathbf{R}^{4}$ ) result as given below $\|1\|$.

2) $E$ is of type $\mathcal{J}_{2}(4)$ : By the trace condition, $E=J(3,0)+J(1,0)$, thus [5], Lemma 3.2 applies with $n=4$ and $\gamma=-3 h_{0}$.

2.1) Subcase: $h_{0} \neq 0$ : By T-design we can assume both translation parts of $\mathbf{E}, \mathbf{H}$ to be zero, and as in [5], D 2), we arrive at the normal forms displayed in $\|2\|$. The three pencils of $\mathbf{E}, \mathbf{H}$ for $\varepsilon \in\{0,1,-1\}$ are not similar, since the corresponding linear pencils are not similar. And the latter is true because the linear pencils generate orbits which are not equiaffinely equivalent. Analogously, the rays of $\mathbf{E}, \mathbf{H}$ are characterized in their pencil, since this is true for the rays of $E, H$ by their Jordan types.

Such characterizations of generators are important for the discussion of similarity by (2.2) or (2.3). For in these systems we then have a priori knowledge on the coefficient scheme. Here, since both rays are unique, the coefficient scheme in (2.3) must be diagonal, 
and with this we can solve this system explicitly resulting in the following self-similarities:

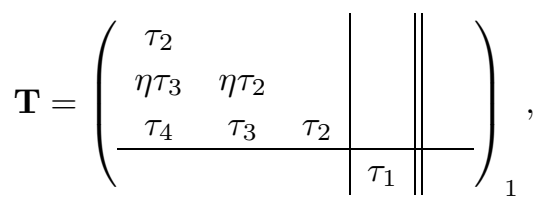

with $\eta \in\{1,-1\}$ and $\tau_{1}, \ldots, \tau_{4}$ free such that $\operatorname{det} \mathbf{T}=\tau_{1} \tau_{2}^{3} \neq 0$.

From this we infer that two points $x$ and $y$ are equivalent under the group of these $\mathbf{T}$ with $|\operatorname{det} \mathbf{T}|=1$ iff $\left|y_{1}^{3} y_{4}\right|=\left|x_{1}^{3} x_{4}\right|$. Thus the corresponding equivalence classes of initial points are classified by $c>0$ with $x_{1}^{6} x_{4}^{2}=c^{2}$. As representative initial points we may choose $(1,0,0, c)^{\top} \in \mathbf{R}^{4}$. Then the orbit parametrization can be calculated by (3.1), and from this follow the implicit orbit equations as announced. Since there are no translation parts in $\mathbf{E}, \mathbf{H}$ the result is the same as in [5], Table for Theorem G, line D I.

2.2) Subcase: $h_{0}=0$ : This case is more difficult. We argue in some detail in order to show how the principles developed above also work in such situations. By T-design and [5], Lemma 3.3, we can start with

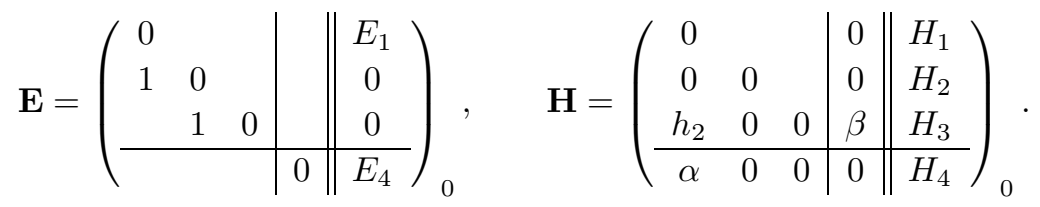

$[\mathbf{E}, \mathbf{H}]=0$ gives $H_{1}=0, H_{2}=h_{2} E_{1}+\beta E_{4}, \alpha E_{1}=0$. By the regularity of the premetric: $E_{1} \neq 0, \beta \neq 0$, hence $\alpha=0$, and then $H_{4} \neq 0$. Another T-design allows $H_{3}=0$. Then rescaling $\mathbf{H}$ with $\frac{1}{\beta}$ yields $\beta=1$. Using an $\mathrm{N}$-design, namely $\mathbf{T}=\operatorname{diag}\left(r^{2}, r, 1,1,1\right)$ with $r:=E_{1}^{-1 / 3}$, shows that $r \mathbf{E}$ and $\mathbf{H}$ may be reduced to

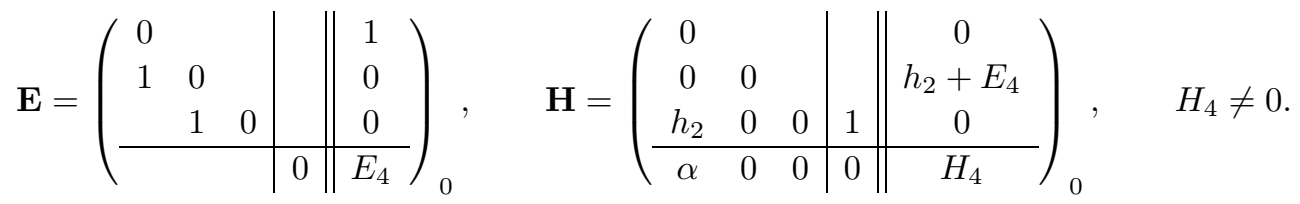

By transforming $\mathbf{E}, \mathbf{H}$ to $\mathbf{E}^{\prime}, \mathbf{H}^{\prime}$ according to

$$
\mathbf{T E}^{\prime} \mathbf{T}^{-1}=c \mathbf{E}-\frac{E_{4}+h_{2}}{c^{2}} \mathbf{H}, \quad \mathbf{T H}^{\prime} \mathbf{T}^{-1}=\mathbf{H} \quad \text { with } \quad c:=\sqrt[3]{H_{4}},
$$

where

$$
\mathbf{T}:=\left(\begin{array}{cccc}
c & 0 & 0 & 0 \\
-\left(\frac{E_{4}+h_{2}}{c}\right)^{2} & c^{2} & 0 & E_{4}+h_{2} \\
0 & -\frac{\left(E_{4}+h_{2}\right)^{2}}{c} & c^{3} & 0 \\
-h_{2} c & 0 & 0 & c^{3}
\end{array}\right)_{1},
$$

we finally obtain $\mathbf{E}^{\prime}, \mathbf{H}^{\prime}$ in the normal form announced in $\|3\|$. (Of course, we then skip the prime. Analogously, in further cases newly constructed generators will obtain the same names without explicit mention.) 
All elements in the pencil of $\mathbf{E}, \mathbf{H}$ are nilpotent of degree 4, except for $\mathbf{H}$ which is of degree 3, thus its ray is unique. Moreover $\mathbf{E}$ is the only element in the pencil whose linear part annihilates the translation part of $\mathbf{H}$, thus its ray is also unique. Knowing this, again the system (2.3) for the self-similarities can be solved, and from the resulting $\mathbf{T}$ one reads off that they operate transitively on all points of $\mathbf{R}^{4}$, hence the representative initial point $(0,0,0,0)^{\top}$.

3) $E$ is of type $\mathcal{J}_{3}(4)$ : By the trace condition, $E=J(2,0)+J(2,0)$. As in [5], D, case 3 ), the most general matrix $H$ commuting with $E$ and of trace 0 and its characteristic polynomial are given by

$E=\left(\begin{array}{cc|cc}0 & & & \\ 1 & 0 & & \\ \hline & 0 & \\ & 1 & 0\end{array}\right), \quad H=\left(\begin{array}{cc|cc}h_{1} & & h_{5} & \\ h_{2} & h_{1} & h_{6} & h_{5} \\ \hline h_{7} & & -h_{1} & \\ h_{8} & h_{7} & h_{4} & -h_{1}\end{array}\right), \quad \chi_{H}(\xi)=\left(\xi^{2}-h_{1}^{2}-h_{5} h_{7}\right)^{2}$.

3.1) Subcase: $h_{1}^{2}+h_{5} h_{7}<0$ : Here $H$ has two purely imaginary double eigenvalues, hence is of type $\mathcal{K}_{1}(4)$ or $\mathcal{K}_{2}(4)$ to be treated in the later cases 10), 11).

3.2) Subcase: $h_{1}^{2}+h_{5} h_{7}>0$ : The discussion in [5] shows that we are left with the situation $E=\operatorname{diag}(1,1,-1,-1)$. No matter how these $E, H$ are completed by translation parts, the premetric becomes singular, so this case doesn't exist here.

3.3) Subcase: $h_{1}^{2}+h_{5} h_{7}=0$ : In [5] this case didn't exist; here the situation is rather complex. We proceed as follows:

3.3.1) Subcase: $h_{1} \neq 0$ : Then $h_{5} h_{7} \neq 0$, and we may assume $h_{5}=1, h_{7}=-h_{1}^{2}$. By TC-design we obtain $E_{2}=0, E_{3}:=-h_{1} E_{1}, E_{4}=0, H_{2}=0$. From the premetric follows $E_{1} \neq 0$, and by forming $\mathbf{E}-h_{2} \mathbf{H}$ we see that $h_{2}=0$ may also be assumed.

Clearly $H$ is nilpotent of degree $\leq 4$. By calculating the powers of $H$ we deduce that the degree is 4 if $h_{1} h_{4}+h_{1}^{2} h_{6}-h_{8} \neq 0$; then we are in case 1$)$. Thus it suffices to consider $h_{8}=h_{1} h_{4}+h_{1}^{2} h_{6}$. Again one sees that the degree is 3 if $h_{4} \neq 0$; then we are in case 2). Thus it suffices to consider $h_{4}=0$. Then $h_{8}=h_{1}^{2} h_{6}$, and the premetric comes out as 0 . So this subcase doesn't occur.

3.3.2) Subcase: $h_{1}=0$ : Then $h_{5} h_{7}=0$, and by symmetry we may assume $h_{5}=0$. If $h_{6} h_{7} \neq 0$ the $H$ is nilpotent of degree 4 , and we are back in case 1 ). So we can assume $h_{6} h_{7}=0$. Then if $h_{4} h_{7} \neq 0$, the degree is 3 , and we are back in case 2). Thus we can assume $h_{4} h_{7}=0$. The commuting gives $E_{1} h_{7}=0$. Again, by forming $\mathbf{E}-h_{2} \mathbf{H}$ we may assume $h_{2}=0$. If $h_{7} \neq 0$ then it follows $h_{4}=h_{6}=E_{1}=0$, and the premetric vanishes.

We are left with $h_{7}=0$. The premetric requires $h_{4}^{2}+4 h_{6} h_{8} \neq 0$, and $H$ is nilpotent of degree 2. If $H$ has rank 1 we enter the later case 4 ). Thus we have only to consider the rank 2 case which means $h_{6} h_{8} \neq 0$.

This preliminary reduction ends, assuming still $h_{8}=1$ by rescaling $H$, with

$$
E=\left(\begin{array}{cc|cc}
0 & & & \\
1 & 0 & & \\
\hline & 0 & \\
& 1 & 0
\end{array}\right), \quad H=\left(\begin{array}{c|c|cc} 
& & 0 & \\
& & h_{6} & 0 \\
\hline 0 & & 0 & \\
1 & 0 & h_{4} & 0
\end{array}\right), \quad h_{6} \neq 0 .
$$


In addition we may assume $\frac{h_{4}^{2}}{4}+h_{6}<0$. The reason is the following: Searching through the pencil elements $E+t H$ for a copy of rank 1 leads to the quadratic equation $h_{6} t^{2}-h_{4} t-1$ $=0$. This has real solutions iff $\frac{h_{4}^{2}}{4}+h_{6} \geq 0$. But then we are again in the later case 4 ).

The further reduction of this linear pencil is missing in [5] because it violates the subspace condition there. So we must do it here. It requires a similarity change in the pencil of $E, H$ which is not so obvious. We found the following solutions of the similarity equations

$$
T E^{\prime} T^{-1}=\alpha_{1} E+\alpha_{2} H, \quad T H^{\prime} T^{-1}=\beta_{1} E+\beta_{2} H:
$$

With

let us set

$$
W:=\sqrt{-\frac{h_{4}^{2}}{4}-h_{6}}
$$

$$
\alpha_{1}:=1, \quad \alpha_{2}:=0, \quad \beta_{1}:=-\frac{h_{4}}{2 W}, \quad \beta_{2}:=\frac{1}{W} .
$$

Then we obtain

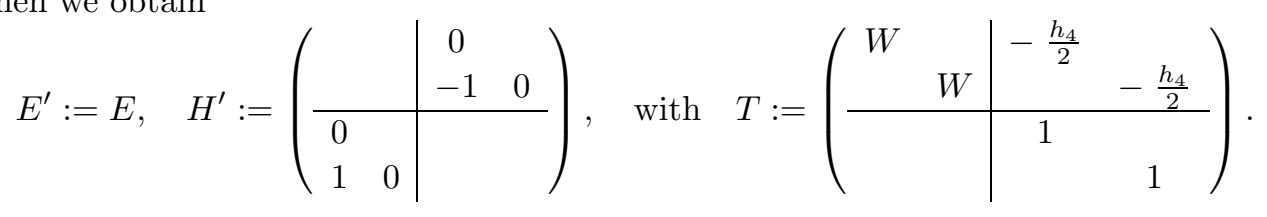

There are no Jordan type properties which can distinguish these two generators: all elements in the pencil of $E, H$ have Jordan normal form $J(2,0)+J(2,0)$. So in this case we investigate the possible uniqueness of generators via the self-similarities $T$, defined as the solutions of

$$
T E T^{-1}=\alpha_{1} E+\alpha_{2} H, \quad T H T^{-1}=\beta_{1} E+\beta_{2} H, \quad \alpha_{1} \beta_{2}-\alpha_{2} \beta_{1} \neq 0 .
$$

The calculation leads to four types, namely two with $\alpha_{2}=\beta_{1}=0$ and $\beta_{2}= \pm \alpha_{1} \neq 0$, thus leaving the rays of $E, H$ invariant:

$$
T_{11}:=\left(\begin{array}{cccc}
\frac{\tau_{1}}{\alpha_{1}} & 0 & -\frac{\tau_{3}}{\alpha_{1}} & 0 \\
\tau_{6} & \tau_{1} & \tau_{5} & -\tau_{3} \\
\frac{\tau_{3}}{\alpha_{1}} & 0 & \frac{\tau_{1}}{\alpha_{1}} & 0 \\
\tau_{4} & \tau_{3} & \tau_{2} & \tau_{1}
\end{array}\right), \quad T_{12}:=\left(\begin{array}{cccc}
-\frac{\tau_{1}}{\alpha_{1}} & 0 & \frac{\tau_{3}}{\alpha_{1}} & 0 \\
\tau_{6} & -\tau_{1} & \tau_{5} & \tau_{3} \\
\frac{\tau_{3}}{\alpha_{1}} & 0 & \frac{\tau_{1}}{\alpha_{1}} & 0 \\
\tau_{4} & \tau_{3} & \tau_{2} & \tau_{1}
\end{array}\right)
$$

and another two with $\alpha_{1}=\beta_{2}=0, \beta_{1}= \pm \alpha_{2} \neq 0$, thus interchanging the spans of $E, H$ :

$$
T_{21}:=\left(\begin{array}{cccc}
\frac{\tau_{3}}{\alpha_{2}} & 0 & \frac{\tau_{1}}{\alpha_{2}} & 0 \\
\tau_{6} & -\tau_{1} & \tau_{5} & \tau_{3} \\
\frac{\tau_{1}}{\alpha_{2}} & 0 & -\frac{\tau_{3}}{\alpha_{2}} & 0 \\
\tau_{4} & \tau_{3} & \tau_{2} & \tau_{1}
\end{array}\right), \quad T_{22}:=\left(\begin{array}{cccc}
\frac{\tau_{3}}{\alpha_{2}} & 0 & \frac{\tau_{1}}{\alpha_{2}} & 0 \\
\tau_{6} & \tau_{1} & \tau_{5} & -\tau_{3} \\
-\frac{\tau_{1}}{\alpha_{2}} & 0 & \frac{\tau_{3}}{\alpha_{2}} & 0 \\
\tau_{4} & \tau_{3} & \tau_{2} & \tau_{1}
\end{array}\right)
$$

where in each case $\left(\tau_{1}, \tau_{3}\right) \neq(0,0)$. The completion by translation parts and TC-design gives at first:

$$
\mathbf{E}=\left(\begin{array}{cc|cc||c}
0 & & & & E_{1} \\
1 & 0 & & & 0 \\
\hline & 0 & & E_{3} \\
& 1 & 0 & 0
\end{array}\right), \quad \mathbf{H}=\left(\begin{array}{cc|cc||c} 
& & 0 & & -E_{3} \\
& -1 & 0 & H_{2} \\
\hline 0 & & & E_{1} \\
1 & 0 & & & H_{4}
\end{array}\right), \quad\left(E_{1}, E_{3}\right) \neq(0,0),
$$

the latter coming from the regularity of the premetric. 
For further reduction, we may apply the pure linear self-similarities from above, say the $T_{11}$ with $\alpha_{1}=1$, taking the translation parts along with them. The new translation parts are then

$$
\left(\begin{array}{c}
\tau_{1} E_{1}-\tau_{3} E_{3} \\
\tau_{6} E_{1}+\tau_{5} E_{3} \\
\tau_{3} E_{1}+\tau_{1} E_{3} \\
\tau_{4} E_{1}+\tau_{2} E_{3}
\end{array}\right), \quad\left(\begin{array}{c}
-\tau_{1} E_{3}-\tau_{3} E_{1} \\
-\tau_{6} E_{3}+\tau_{1} H_{2}+\tau_{5} E_{1}-\tau_{3} H_{4} \\
\tau_{1} E_{1}-\tau_{3} E_{3} \\
-\tau_{4} E_{3}+\tau_{3} H_{2}+\tau_{2} E_{1}+\tau_{1} H_{4}
\end{array}\right)
$$

In order to maintain the old form we must have $\tau_{6} E_{1}+\tau_{5} E_{3}=\tau_{4} E_{1}+\tau_{2} E_{3}=0$, which means $\left(\tau_{5}, \tau_{6}\right)=r\left(E_{1},-E_{3}\right),\left(\tau_{2}, \tau_{4}\right)=s\left(E_{1},-E_{3}\right)$ for some $r, s$. In addition we can reach $\tau_{3} E_{1}+\tau_{1} E_{3}=0$, i.e. $\left(\tau_{1}, \tau_{3}\right)=u\left(E_{1},-E_{3}\right)$ for some $u \neq 0$. Then the new translation parts become

$$
\left(\begin{array}{c}
u\left(E_{1}^{2}+E_{3}^{2}\right) \\
0 \\
0 \\
0
\end{array}\right), \quad\left(\begin{array}{c}
0 \\
r\left(E_{1}^{2}+E_{3}^{2}\right)+u\left(E_{1} H_{2}+E_{3} H_{4}\right) \\
u\left(E_{1}^{2}+E_{3}^{2}\right) \\
s\left(E_{1}^{2}+E_{3}^{2}\right)+u\left(E_{1} H_{4}-E_{3} H_{2}\right)
\end{array}\right),
$$

hence we can finally reach the translation parts $(1,0,0,0)^{\top}$ and $(0,0,1,0)^{\top}$, as announced in $\|4\|$.

Now the self-similarities $\mathbf{T}$ for the pencil of $\mathbf{E}, \mathbf{H}$ can easily be computed, since their linear parts are known from above for the linear parts $E, H$. The result is that the $\mathbf{T}$ with $|\operatorname{det} \mathbf{T}|=1$ operate transitively on the points of $\mathbf{R}^{4}$, hence the representative initial point $(0,0,0,0)^{\top}$. The orbit parametrization and the implicit equations are then straightforward.

4) $E$ is of type $\mathcal{J}_{4}(4)$ : This linear case is also missing in [5] because the centro-affine metric degenerates, so again we have to do it here.

By the trace condition $E=J(2,0)+J(1,0)+J(1,0)$. The most general $H$ commuting with $E$ is

$$
H:=\left(\begin{array}{cc|cc}
h_{9} & 0 & 0 & 0 \\
h_{10} & h_{9} & h_{8} & h_{7} \\
\hline h_{6} & 0 & h_{5} & h_{4} \\
h_{3} & 0 & h_{2} & h_{1}
\end{array}\right)
$$

It has the characteristic polynomial

$$
\chi_{H}(\xi):=\left(\xi-h_{9}\right)^{2}\left(\xi^{2}-\left(h_{1}+h_{5}\right) \xi+h_{1} h_{5}-h_{2} h_{4}\right)
$$

with the roots

$$
h_{9} \text { double } \quad \text { and } \quad \xi_{1,2}:=-\frac{h_{9}}{2} \pm \sqrt{W}, \quad W:=\frac{\left(h_{1}-h_{5}\right)^{2}}{4}+h_{2} h_{4} .
$$

If $W<0$ then we are in a later complex case.

4.1) Subcase: $W>0$ and $h_{9}$ is distinct from the other two roots: Then either the generalized eigenspace of $H$ for the eigenvalue $h_{9}$ does not split under $H$, which leads to the later case $\mathcal{J}_{1}(2)+\mathcal{J}_{1}(1)+\mathcal{J}_{1}^{\prime}(1)$, i.e. 9$)$, for $H$, or it splits, and then the eigenspaces of $H$ span $\mathbf{R}^{4}$ :

$$
\mathbf{R}^{4}=E_{H}\left(h_{9}\right) \oplus E_{H}\left(\xi_{1}\right) \oplus E_{H}\left(\xi_{2}\right), \quad \operatorname{dim} E_{H}\left(h_{9}\right)=2 .
$$


By the commuting of $E, H$, the eigenspaces of $H$ are invariant under $E$. This forces $E$ to be zero on the last two eigenspaces and to have Jordan normal form $J_{1}(2)$ on the first one. Thus we obtain the simultaneous normal forms

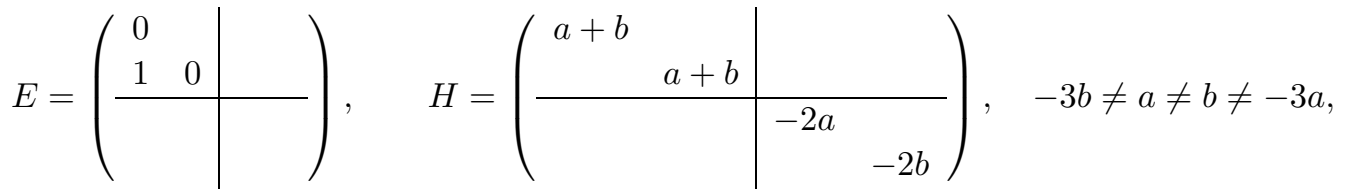

where we changed the notation for the eigenvalues of $H$ appropriately, in view of the trace condition.

For the affine generators, we reach by TC-design:

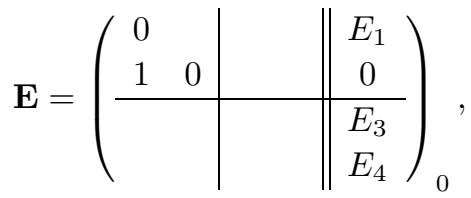

$$
\mathbf{H}=\left(\begin{array}{cc|cc||c}
a+b & & & \\
& a+b & & & \\
H_{2} \\
\hline & & -2 a & & H_{3} \\
H_{4}
\end{array}\right)_{0}
$$

with $(a+b) E_{1}=2 a E_{3}=2 b E_{4}=0$. From the premetric we deduce $E_{1} \neq 0$ and thus $a+b=0, a \neq 0$ and $E_{3}=E_{4}=0$. A T-design then yields $H_{3}=H_{4}=0$. We can rescale $\mathbf{H}$ to obtain $2 a=-1$ and by a pure linear similarity of the form $\operatorname{diag}\left(r^{2} s, r s^{2}, 1,1,1\right)$ (mixed N-designs) we may additionally reach $E_{1}=1$ and $H_{2}=\varepsilon \in\{0,1,-1\}$. The result is as announced in $\|5\|$. The linear parts generate distinguished rays because $E$ is the only nilpotent and $H$ is the only diagonalizable element in the pencil. Thus also the spans of $\mathbf{E}, \mathbf{H}$ are unique. From this the calculation of self-similarities is straightforward, and it leads to the representative initial points as advertised below $\|5\|$.

4.2) Subcase: $W>0$ and $h_{9}$ coincides with one of the other two roots, say $\xi_{1}$ : Then either $H$ is of type $\mathcal{J}_{1}(3)+\mathcal{J}_{1}(1)$ or $\mathcal{J}_{2}(3)+\mathcal{J}_{1}(1)$, leading to the later cases 5$), 6$ ), or else $H$ is diagonalizable: $\mathbf{R}^{4}=E_{H}\left(h_{9}\right) \oplus E_{H}\left(\xi_{2}\right)$, $\operatorname{dim} E_{H}\left(h_{9}\right)=3$. By the same arguments as above we can reach $E=J(2,0)+J(1,0)+J(1,0), H=\operatorname{diag}(a, a, a,-3 a)$ with $a \neq 0$. By T-design both translation parts can be made zero. But then the premetric turns out to be 0 , so this case doesn't exist here.

4.3) Subcase: $W=0$ : Then $h_{9}$ is a double root and also $\frac{h_{9}}{2}$, so by the trace condition $h_{9}=0$, and $H$ is nilpotent. If its Jordan type is $\mathcal{J}_{1}(4)$ or $\mathcal{J}_{2}(4)$ we are in the former cases 1) or 2). So we can assume the Jordan type of $H$ to be $\mathcal{J}_{3}(4)$ or $\mathcal{J}_{4}(4)$, i.e. $H^{2}=0$ and $H \neq 0$.

Without loss of generality we may assume $h_{10}=0$ (by subtracting from $H$ a multiple of $E$ ) and also $h_{5}=-h_{1}$ by $h_{9}=0$ and the trace condition. Looking at $H^{2}=0$ we deduce that the last two columns of $H$ are linearly dependent. By possibly interchanging the last two base vectors, we obtain the following form for $H$ :

$$
H:=\left(\begin{array}{cc|cc} 
& & 0 & 0 \\
& & h_{8} & r h_{8} \\
\hline h_{6} & 0 & -r h_{2} & -r^{2} h_{2} \\
h_{3} & 0 & h_{2} & r h_{2}
\end{array}\right) \quad \text { for some } r \in \mathbf{R} .
$$

Changing the last base vector according to $e_{4}^{\prime}:=-r e_{3}+e_{4}$ allows us to assume $r=0$, 
where $h_{8} h_{6}=h_{2} h_{6}=0$ from the commuting. Another similarity of the form

$$
T:=\left(\begin{array}{cc|cc}
1 & & 0 & 0 \\
& 1 & 0 & t_{7} \\
\hline t_{6} & 0 & 1 & \\
0 & 0 & & 1
\end{array}\right)
$$

also leaves $E$ invariant and changes $H$ to

$$
T H T^{-1}=\left(\begin{array}{cc|cc}
0 & 0 & \\
t_{7} h_{3}-t_{6}\left(h_{8}+t_{7} h_{2}\right) & 0 & h_{8}+t_{7} h_{2} & 0 \\
\hline h_{6} & 0 & 0 & \\
h_{3}-h_{2} t_{6} & 0 & h_{2} & 0
\end{array}\right) .
$$

4.3.1) Subcase: $h_{2} \neq 0$ : Solving $h_{8}+t_{7} h_{2}=h_{3}-h_{2} t_{6}=0$ for $t_{7}, t_{6}$ and subtracting from the new $H$ a multiple of $E$ reduces $H$ to the form as in the announcement $\|6\|$, where $h_{6}=0$ follows from $h_{2} h_{6}=0$ and $h_{2}=1$ by rescaling. All elements in the pencil of $E, H$ are nilpotent of degree 2. The two spans of $E, H$ are the only ones which are of rank 1, so uniquely determined, however interchangeable. For the affine generators, we reach by TC-design $E_{2}=E_{3}=H_{1}=H_{4}=0$ with $E_{1} \neq 0, H_{3} \neq 0$ from the premetric. Moreover, by N-design we can assume $E_{1}=H_{3}=1$, and then by a pure linear similarity of the form

$$
T:=\left(\begin{array}{cc|cc}
1 & & 0 & \\
& 1 & t_{8} & 0 \\
\hline 0 & & 1 & \\
t_{3} & 0 & & 1
\end{array}\right)
$$

we can reach $E_{4}=H_{2}=0$, thus $\mathbf{E}, \mathbf{H}$ as advertised in $\|6\|$. Again, the two spans of $\mathbf{E}, \mathbf{H}$ are uniquely determined but equally entitled from which follow the self-similarities and representative initial point as usual.

From now on, when the calculation of self-similarities, representative initial points and orbit representations are straightforward, we do not mention this further, but only display the result in the announcement.

4.3.2) Subcase: $h_{2}=0$ : Here we have from the above

$$
H:=\left(\begin{array}{cc|cc} 
& & 0 & \\
& & h_{8} & 0 \\
\hline h_{6} & 0 & & \\
h_{3} & 0 & &
\end{array}\right) \quad \text { with } \quad h_{8} h_{6}=0 .
$$

For the affine generators, we reach by TC-design $E_{2}=0, H_{1}=h_{8} E_{3}$ with $h_{6} E_{1}=$ $h_{3} E_{1}=0$. The discriminant turns out to be 0 under these side conditions, so this case doesn't exist.

5) $E$ is of type $\mathcal{J}_{1}(3)+\mathcal{J}_{1}(1)$ : By the trace condition, $E=J(3, a)+J(1,-3 a)$ with real $a \neq 0$. The most general matrix commuting with $E$ and of trace 0 is as follows: 


$$
E=\left(\begin{array}{ccc|c}
a & & & \\
1 & a & & \\
& 1 & a & \\
\hline & & & -3 a
\end{array}\right), \quad H=\left(\begin{array}{ccc|c}
h_{0} & & & \\
h_{1} & h_{0} & & \\
h_{2} & h_{1} & h_{0} & \\
\hline & & & -3 h_{0}
\end{array}\right) .
$$

By subtracting from $H$ a multiple of $E$, we may assume $h_{0}=0$. After T-design the translation parts of $\mathbf{E}, \mathbf{H}$ are 0 . From the the premetric follows $h_{1} \neq 0$, and this implies that $H$ is nilpotent of degree 3 . Hence with $H$ we are in the former case 2).

6) $E$ is of type $\mathcal{J}_{2}(3)+\mathcal{J}_{1}(1)$ : By the trace condition $E=J(2,1, a)+J(1,-3 a)$ with real $a \neq 0$. The most general matrix $H$ commuting with $E$ is as follows:

$$
E=\left(\begin{array}{ccc|c}
a & & & \\
1 & a & & \\
& & a & \\
\hline & & & -3 a
\end{array}\right), \quad H=\left(\begin{array}{ccc|c}
h_{0} & & 0 & \\
h_{1} & h_{0} & \beta & \\
\alpha & 0 & \gamma & \\
\hline & & & h_{2}
\end{array}\right) .
$$

In the generalized eigenspace of $E$ for the eigenvalue $a$ we have the situation of [5], Lemma 3.2. So there are two natural subcases:

If $\gamma \neq h_{0}$ then we can reach $\alpha=\beta=0$. By subtracting from $H$ a multiple of $E$, we obtain a diagonalizable new $H$, i.e. we can reach $h_{1}=0$. In case $h_{0} \neq 0$, by subtracting from $E$ a multiple of $H$, the new $E$ becomes $J(2,0)+\operatorname{diag}(b,-b)$, with $b \neq 0$; this $E$ is of type $\mathcal{J}_{1}(2)+\mathcal{J}_{1}(1)+\mathcal{J}_{1}^{\prime}(1)$ and will be treated in the later case 9$)$. In case $h_{0}=0$, by adding a suitable multiple of $H$ to $E$, this becomes of type $\mathcal{J}_{1}(2)+\mathcal{J}_{2}(2)$, so this situation will be included in the later case 8 ).

If $\gamma=h_{0}$ then, by subtracting from $H$ a suitable multiple of $E$, we may assume $h_{0}=0$. After T-design both translation parts are 0 . The premetric requires $\alpha \beta \neq 0$, and then $H$ is nilpotent of degree 3, so belongs to case 2).

7) $E$ is of type $\mathcal{J}_{1}(2)+\mathcal{J}_{1}^{\prime}(2)$ : By the trace condition $E=J(2, a)+J(2,-a)$ with real $a \neq 0$. The most general matrix commuting with $E$ and of trace 0 is as follows:

$$
E=\left(\begin{array}{cc|cc}
a & & \\
1 & a & & \\
\hline & & -a & \\
& 1 & -a
\end{array}\right), \quad H=\left(\begin{array}{cc|cc}
h_{0} & & & \\
h_{1} & h_{0} & & \\
\hline & & -h_{0} & \\
& & h_{2} & -h_{0}
\end{array}\right) .
$$

By subtracting from $H$ a multiple of $E$ we may assume $h_{0}=0$. Again after T-design both translation parts vanish, and then the premetric turns out to be of rank $\leq 1$.

8) $E$ is of type $\mathcal{J}_{1}(2)+\mathcal{J}_{2}(2)$ : By the same arguments as in [5], we can assume

$$
E=\left(\begin{array}{ll|ll}
1 & & & \\
1 & 1 & & \\
\hline & -1 & \\
& & & -1
\end{array}\right), \quad H=\left(\begin{array}{ll|ll}
h_{0} & & & \\
& h_{0} & & \\
\hline & & h_{2} & h_{3} \\
& & h_{4} & h_{5}
\end{array}\right) .
$$

and the following subcases follow the type of the lower-right box of $H$ :

The lower-right box of $H$ is of type $\mathcal{J}_{1}(2)$ : We may reach $H=\operatorname{diag}(-b,-b)+J(2, b)$. Then both translation parts can be set to 0 , and the premetric is calculated to be of rank $\leq 1$. 
The lower-right box of $H$ is $\mathbf{R}$-diagonalizable, thus $h_{3}=h_{4}=0$ is available. Again, both translation parts can be set to 0 , and the premetric requires $h_{0}+h_{2} \neq 0$. As in [5], case $\mathrm{D} 8$ ), there is an element in the linear pencil of type $\mathcal{J}_{1}(2)+\mathcal{J}_{1}(1)+\mathcal{J}_{1}^{\prime}(1)$, to be treated in the next case 9).

The lower-right box of $H$ is $\mathbf{C}$-diagonalizable (genuine), so this is one of the later complex cases.

9) $E$ is of type $\mathcal{J}_{1}(2)+\mathcal{J}_{1}(1)+\mathcal{J}_{1}^{\prime}(1)$ : Then $E=J(2, a)+\operatorname{diag}(\beta, \gamma)$ with mutually different $a, \beta, \gamma$. The most general matrix $H$ commuting with $E$ is given as follows:

$$
E=\left(\begin{array}{cc|cc}
a & & & \\
1 & a & & \\
\hline & & \beta & \\
& & & \gamma
\end{array}\right), \quad H=\left(\begin{array}{ll|ll}
h_{0} & & & \\
h_{1} & h_{0} & & \\
\hline & & h_{2} & \\
& & & h_{3}
\end{array}\right),
$$

where we may reach $h_{1}=0$ by subtracting a multiple of $E$.

9.1) Subcase: $h_{0} \neq 0$ : We may assume $h_{0}=1$ and, by subtracting from $E$ a multiple of $H$, moreover $a=0$. Thus $E=J(2,0)+\operatorname{diag}(\beta,-\beta)$. By TC-design: $E_{1}=E_{2}=H_{1}=$ $H_{2}=0$. Then the premetric yields $\beta \neq 0$ thus $\beta=1$. Another TC-design then allows $E_{3}=E_{4}=H_{3}=E_{4}=0$. With $b:=h_{2}$ we thus arrive at $\mathbf{E}, \mathbf{H}$ as announced in $\|7\|$. The premetric gives no further restriction on $b$. Regarding this, we have the same situation as in [5]: If we replace $b$ by $-2-b$ then the similarity class of $\operatorname{span}(E, H)$ does not change because $E$ may be multiplied by -1 , and the last two base vectors may be interchanged. So we may assume $b \geq-1$. Exactly the elements of $\operatorname{span}(H)$ are $\mathbf{R}$-diagonalizable. All other rays in $\operatorname{span}(E, H)$ are of type $\mathcal{J}_{1}(2)+\mathcal{J}_{1}(1)+\mathcal{J}_{1}^{\prime}(1)$, up to one exception of type $\mathcal{J}_{2}(3)+\mathcal{J}_{1}(1)$, and among them exactly $\operatorname{span}(E)$ has double eigenvalue 0 . The rank of $H$ is always $\geq 3$. From the spectrum of $H$ one deduces that if $b \geq-1$ and $b^{\prime} \geq-1$, the corresponding pencils $\operatorname{span}(E, H), \operatorname{span}\left(E, H^{\prime}\right)$ are similar iff $b=b^{\prime}$.

9.2) Subcase: $h_{0}=0$ : If we had $a=0$ then we could reach $h_{2}=-h_{3}=1$; but then $E-\beta G$ would be of type $\mathcal{J}_{4}(4)$, already treated in case 4$)$. So we can assume $a \neq 0$ and achieve the same normal forms for $E, H$ as in [5]. By C-design we obtain the normal forms $\|8\|$. The rays of $E$ and $H$ are the only ones in their pencil whose elements are of type $\mathcal{J}_{1}(2)+\mathcal{J}_{1}(1)+\mathcal{J}_{1}^{\prime}(1)$ and have a single eigenvalue 0 . As a consequence, the rays of $\mathbf{E}$ and $\mathbf{H}$ are unique but may be interchanged.

So far, all elements of the linear pencils belonging to the cases $\|1\|$ to $\|8\|$ have real spectra, and the inclusions are non-circular.

10) $E$ is of type $\mathcal{K}_{1}(4)$ : For $E, H$ there is the same reduction possible as in [5], D 10), and since $E$ is nondegenerate there are no translation parts, by T-design. But then the premetric turns out to have rank $\leq 1$. This is also clear from the fact that the orbits of $\mathbf{H}$ are straight, and ruled surfaces can never occur as nondegenerate surfaces.

11) $E$ is of type $\mathcal{K}_{2}(4)$ : The discussion for $E, H$ is the same as in [5], D 11), till the point where the type (VI) there is reached (up to some diagonalizable cases for $H$ to be delegated). Again both translation parts will vanish after T-design, and then the orbits of $\mathbf{H}$ are straight again, so this case doesn't occur either. 
12) $E$ is of type $\mathcal{J}_{1}(2)+\mathcal{K}_{1}(2)$ : As in [5] we can reach the following $E$ with the given $H$ as the most general matrix of trace 0 commuting with $E$ :

$$
E=\left(\begin{array}{cc|cc}
\alpha & & \\
1 & \alpha & & \\
\hline & & -\alpha & -\beta \\
& \beta & -\alpha
\end{array}\right), \quad \beta>0, \quad H=\left(\begin{array}{cc|cc}
h_{0} & & & \\
h_{1} & h_{0} & & \\
\hline & & -h_{0} & -h_{2} \\
& & h_{2} & -h_{0}
\end{array}\right) .
$$

We can assume $h_{1}=0$ by subtracting $h_{1} E$ from $H$.

In case $h_{0}=0$ the $H$ becomes of type $\mathcal{J}_{2}(2)+\mathcal{K}_{1}(2)$, so this will be included in the next case 13).

If $h_{0} \neq 0$ we may additionally assume $h_{0}=1$ by rescaling $H$, then subtract $\alpha H$ from $E$ in order to reach $\alpha=0$. The $H$ being regular, both translation parts can be made zero, and from the premetric follows $\beta \neq 0$, without loss $\beta>0$ (by possibly interchanging the last two base vectors). Then the diagonal similarity $\mathbf{T}_{0}:=\operatorname{diag}\left(1, \beta, \beta^{-1 / 2}, \beta^{-1 / 2}, 1\right)$ and rescaling $\mathbf{E}$ with $\beta^{-1}$ yield the normal forms as given in $\|9\|$, where the premetric requires $b^{2} \neq 32$. Moreover, interchanging the last two base vectors, multiplying $\mathbf{E}$ by -1 , and applying [5], Lemma 3.3 allows us to assume $b \geq 0$ (and then $b \neq \sqrt{32}$ ).

The rays of $E, H$ in their pencil are unique, since $H$ and its multiples are the only diagonalizable, and $E$ and its multiples are the only nondiagonalizable, singular elements. Obviously, $b \geq 0$ is separating within the ray of $H$.

So the discussion of self-similarities and initial points is straightforward. However there is one additional phenomenon: For $b>0$ the self-similarities come out as:

$$
\mathbf{T}_{1}=\left(\begin{array}{cc|cc||c}
\tau_{3} & & & & \\
\tau_{4} & \tau_{3} & & & \\
\hline & & \tau_{1} & -\tau_{2} &
\end{array}\right)_{1}, \quad \operatorname{det} \mathbf{T}_{1}=\left(\tau_{1}^{2}+\tau_{2}^{2}\right) \tau_{3}^{2} \neq 0
$$

For $b=0$ one also has this group of self-similarities, but another isolated one to adjoin, namely $\operatorname{diag}(1,-1,1,-1,1)$. In some later situations, this phenomenon will also occur. For the initial point discussion this has to be regarded, but causes no difficulties.

13) $E$ is of type $\mathcal{J}_{2}(2)+\mathcal{K}_{1}(2)$ : As in [5], D 13), we may assume the following $E$ with the given $H$ as the most general matrix commuting with $E$ :

$$
E=\left(\begin{array}{cc|cc}
a & & \\
& a & & \\
\hline & -a & -b \\
& b & -a
\end{array}\right), \quad b>0, \quad H=\left(\begin{array}{cc|cc}
h_{1} & h_{2} & & \\
h_{3} & h_{4} & & \\
\hline & & h_{5} & -h_{6} \\
& h_{6} & h_{5}
\end{array}\right) .
$$

We can reach $h_{6}=0$ by subtraction and moreover assume that the upper-left $(2 \times 2)$ block of $H$ is of type $\mathcal{J}_{1}(2)$ because otherwise both $E, H$ are diagonalizable. By the trace condition $H=J\left(2, h_{1}\right)+\operatorname{diag}\left(-h_{1},-h_{1}\right)$.

13.1) Subcase: $h_{1} \neq 0$ : If we had $a \neq 0$, by adding a suitable multiple of $E$ to $H$ and interchanging the role of $E, H$, we would arrive at the situation of case 12), first part. So it remains only $a=0$, and then by [5], Lemma 3.3 we obtain $E, H$ as announced in $\|10\|$, where both translation parts are made zero by T-design and $b$ becomes 1 by rescaling. 
The ray of $E$, resp. $H$ is unique by the property that it contains only diagonalizable elements, resp. elements with real spectrum.

13.2) Subcase: $h_{1}=0$ : If we had $a \neq 0$, then besides $E_{3}=E_{4}=H_{2}=0$ we could reach by TC-design that both translation parts vanish; and then the premetric will vanish. So we must have $a=0$. Then by commuting $E_{1}=H_{3}=H_{4}=0$, and the premetric requires $H_{1} \neq 0$. By H-design we can reach $H_{1}=1$, moreover $b=1$ (by rescaling $\mathbf{E}$ ). By an $\mathrm{N}$-design of the form $\operatorname{diag}\left(s, s^{2}, 1,1,1\right)$ it is possible to reach $E_{2} \in\{0,1,-1\}$ and still by one of the form $\operatorname{diag}(1,1,1,-1,1)$ finally $E_{2}=\varepsilon \in\{0,1\}$, where these two pencils are not similar. Hence the normal forms as advertised in $\|11\|,\|12\|$.

Again the rays of $E$ and $H$ are unique as above.

14) $E$ is of type $\mathcal{K}_{1}(2)+\mathcal{K}_{1}^{\prime}(2):$ As in [5] this can be delegated to a diagonalizable case.

15) $E$ is of type $\mathcal{J}_{1}(1)+\mathcal{J}_{1}^{\prime}(1)+\mathcal{K}_{1}(2)$ : Same as in 14).

4. The commutative diagonalizable case. Here we study the case $[\mathbf{E}, \mathbf{H}]=0$ where the corresponding linear pencils contain only diagonalizable elements. For the generators of the linear pencil we have to consider the following four situations as in [5], Sect. 4:

$$
\begin{array}{c|c|c|c}
\mathrm{I} & \mathrm{II} & \mathrm{III} & \mathrm{IV} \\
E=\operatorname{diag}(N, R) & E=\operatorname{diag}(N, N) & E=\operatorname{diag}(N, R) & E=\operatorname{diag}(R, R) \\
H=\operatorname{diag}(R, R) & H=\operatorname{diag}(R, R) & H=\operatorname{diag}(R, N) & H=\operatorname{diag}(R, R) .
\end{array}
$$

$R$ stands for a real diagonal $(2 \times 2)$-block and $N$ for a normal $(2 \times 2)$-block, not necessarily the same at different places. A normal block has the form

$$
N(\lambda, \mu):=\left(\begin{array}{cc}
\lambda & -\mu \\
\mu & \lambda
\end{array}\right) \quad \text { with } \quad \mu>0 .
$$

B. Theorem. Let $\Gamma^{\bullet}$ be a two-dimensional commutative Lie subalgebra of $\mathbf{S A}(4, \mathbf{R})^{\bullet}$, whose orbits are not all degenerate with respect to the Burstin-Mayer metric. Assume that all elements of the corresponding linear pencil $\Gamma^{\bullet \prime}$ are diagonalizable.

Then $\Gamma^{\bullet}$ is similar to one of the following eight cases $\|13\|, \ldots,\|20\|$, specifying $\Gamma^{\bullet}$ by the given generators $\mathbf{E}, \mathbf{H}$, the initial points $x_{0}$, and the discriminants $\delta$ and implicit equations as in Theorem $A$.

Within each case, the parameters possibly occurring in $\mathbf{E}, \mathbf{H}$, resp. $x_{0}$ are separating under the given conditions between similarity classes of pencils, resp. congruence classes of orbits.

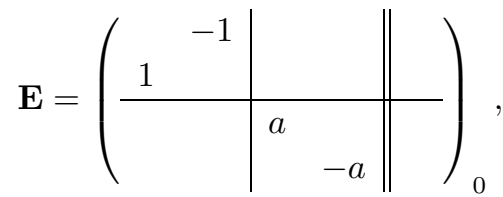

$$
\mathbf{H}=\left(\begin{array}{ll|ll||}
1 & & \\
& 1 & & \\
& & b-1 & \\
& & & -b-1
\end{array}\right)_{0}
$$




$$
\begin{aligned}
& \left\{\begin{array} { l } 
{ a > 0 } \\
{ b \geq 0 } \\
{ a \neq \sqrt { - \lambda \pm \sqrt { \omega } } \quad \text { if } \quad b \leq \frac { 2 } { \sqrt { 3 } } }
\end{array} , \quad \text { where } \left\{\begin{array}{l}
\lambda:=\frac{b^{4}+9 b^{2}-16}{b^{2}+32} \\
\omega:=4 \cdot \frac{\left(b^{2}-4\right)^{2}\left(4-3 b^{2}\right)}{\left(b^{2}+32\right)^{2}}
\end{array}\right.\right. \\
& \quad=\quad-p(a, b)\left(x_{1}^{2}+x_{2}^{2}\right)^{2} x_{3}^{2} x_{4}^{2}, \quad \text { where } \\
& p(a, b) \quad:=\left(b^{2}+32\right) a^{4}+2\left(b^{4}+9 b^{2}-16\right) a^{2}+b^{2}\left(b^{2}-1\right)^{2} \\
& x_{0}=(1,0,1, c)^{\top}, \quad c>0, \quad\left\{\ln x_{3}-2 a \arctan \frac{x_{2}}{x_{1}}-(b-1) \ln \left(x_{1}^{2}+x_{2}^{2}\right)=0 .\right.
\end{aligned}
$$

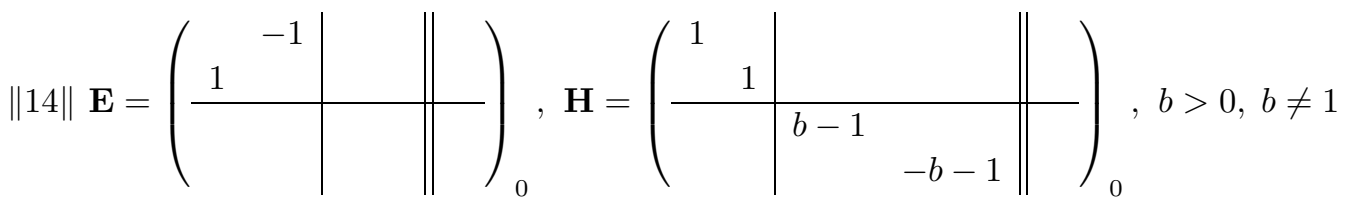

$$
\delta=-b^{2}\left(b^{2}-1\right)^{2}\left(x_{1}^{2}+x_{2}^{2}\right)^{2} x_{3}^{2} x_{4}^{2}
$$

$$
x_{0}=(1,0,1, c)^{\top}, \quad c>0, \quad\left\{\begin{array}{r}
\left(x_{1}^{2}+x_{2}^{2}\right) x_{3} x_{4}=c \\
2 \ln x_{3}-(b-1) \ln \left(x_{1}^{2}+x_{2}^{2}\right)=0 .
\end{array}\right.
$$

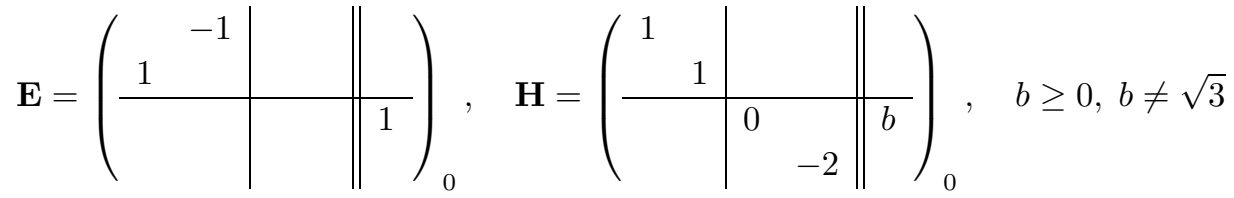

$$
\begin{aligned}
& \delta=4\left(3-b^{2}\right)\left(x_{1}^{2}+x_{2}^{2}\right)^{2} x_{4}^{2} \\
& x_{0}=(1,0,0, c)^{\top}, \quad c>0, \quad\left\{\begin{array}{r}
\left(x_{1}^{2}+x_{2}^{2}\right) x_{4}=c \\
2 x_{3}-2 \arctan \frac{x_{2}}{x_{1}}-b \ln \left(x_{1}^{2}+x_{2}^{2}\right)=0 .
\end{array}\right.
\end{aligned}
$$

(I.4)

$\|16\|$

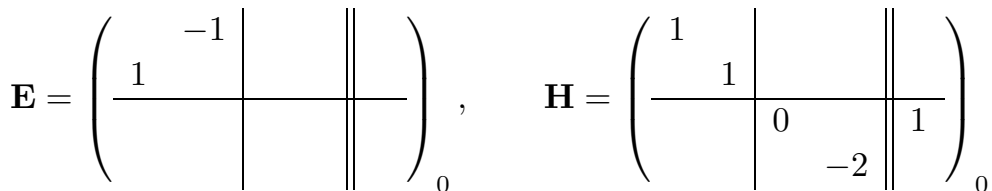

$$
\begin{aligned}
& \delta=-4\left(x_{1}^{2}+x_{2}^{2}\right)^{2} x_{4}^{2} \\
& x_{0}=(1,0,0, c)^{\top}, \quad c>0, \quad\left\{\begin{array}{r}
\left(x_{1}^{2}+x_{2}^{2}\right) x_{4}=c \\
2 x_{3}-\ln \left(x_{1}^{2}+x_{2}^{2}\right)=0 .
\end{array}\right.
\end{aligned}
$$

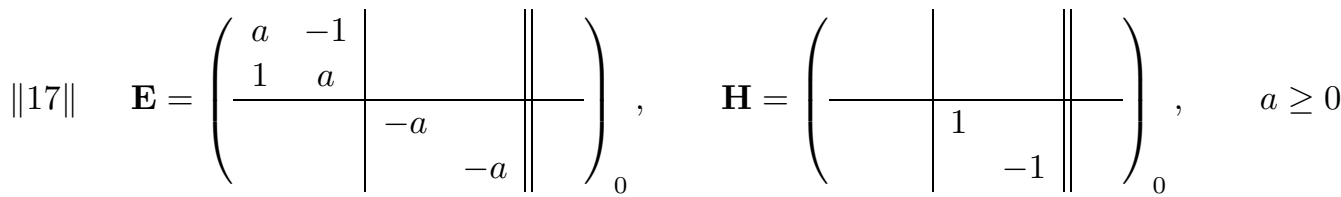




$$
\begin{gathered}
\delta=-\left(a^{2}+1\right)^{2}\left(x_{1}^{2}+x_{2}^{2}\right)^{2} x_{3}^{2} x_{4}^{2} \\
x_{0}=(1,0,1, c)^{\top}, \quad c>0, \quad\left\{\begin{array}{r}
\left(x_{1}^{2}+x_{2}^{2}\right) x_{3} x_{4}=c \\
2 a \arctan \frac{x_{2}}{x_{1}}-\ln \left(x_{1}^{2}+x_{2}^{2}\right)=0 .
\end{array}\right.
\end{gathered}
$$

(II)

$$
\begin{aligned}
& \|18\| \quad \mathbf{E}=\left(\begin{array}{cc|c||c|c}
0 & -1 & & & \\
1 & 0 & & & \\
\hline & & 0 & -a & \\
& & 0 &
\end{array}\right)_{0}, \quad \mathbf{H}=\left(\begin{array}{cc|cc}
1 & & \\
& 1 & & \\
\hline & & -1 &
\end{array}\right)_{0}, \quad 0<a \leq 1 \\
& \delta=8 a^{2}\left(a^{2}+1\right)\left(x_{1}^{2}+x_{2}^{2}\right)^{2}\left(x_{3}^{2}+x_{4}^{2}\right)^{2} \\
& x_{0}=(c, 0,1,0)^{\top}, \quad c>0, \quad\left\{\begin{array}{r}
\left(x_{1}^{2}+x_{2}^{2}\right)\left(x_{3}^{2}+x_{4}^{2}\right)=c^{2} \\
a \arctan \frac{x_{2}}{x_{1}}-\arctan \frac{x_{4}}{x_{3}}=0 .
\end{array}\right.
\end{aligned}
$$

(III)

$$
\begin{aligned}
& \|19\| \quad \mathbf{E}=\left(\begin{array}{cc|c||c|c|c}
a & -1 & & & \\
1 & a & & & \\
\hline & & -a & &
\end{array}\right)_{0}, \quad \mathbf{H}=\left(\begin{array}{ccc|c}
-b & & & \\
& -b & & \\
\hline & & b & -1 \\
& & &
\end{array}\right)_{0} \\
& \left\{\begin{array} { l } 
{ 0 \leq a \leq b } \\
{ a \neq \sqrt { - \lambda + \sqrt { \omega } } \quad \text { if } b > \frac { 1 } { \sqrt { 3 2 } } }
\end{array} , \quad \text { where } \left\{\begin{array}{l}
\lambda:=\frac{16 b^{4}+9 b^{2}-1}{32 b^{2}-1} \\
\omega:=4 \cdot \frac{b^{2}\left(4 b^{2}+3\right)\left(4 b^{2}+1\right)^{2}}{\left(32 b^{2}-1\right)^{2}}
\end{array}\right.\right. \\
& \delta=\frac{1}{4} p(a, b)\left(x_{1}^{2}+x_{2}^{2}\right)^{2}\left(x_{3}^{2}+x_{4}^{2}\right)^{2} \text {, where } \\
& p(a, b):=\left(32 b^{2}-1\right) a^{4}+2\left(16 b^{4}+9 b^{2}-1\right) a^{2}-\left(b^{2}+1\right)^{2} \\
& x_{0}=(c, 0,1,0)^{\top}, \quad c>0, \quad\left\{\begin{array}{r}
\left(x_{1}^{2}+x_{2}^{2}\right)\left(x_{3}^{2}+x_{4}^{2}\right)=c^{2} \\
2 a \arctan \frac{x_{2}}{x_{1}}-2 b \arctan \frac{x_{4}}{x_{3}}-\ln \left(x_{3}^{2}+x_{4}^{2}\right)=0 .
\end{array}\right.
\end{aligned}
$$

(IV)

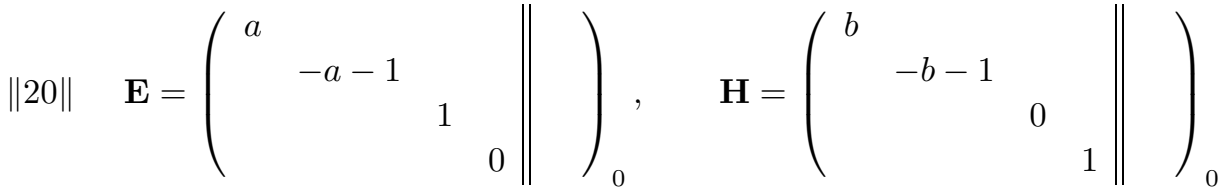

$$
\begin{aligned}
& 0 \leq a \leq b+1 \leq 1, \quad(a, b) \neq(0,0),(1,0) \\
& \delta=-p(a, b) x_{1}^{2} x_{2}^{2} x_{3}^{2} x_{4}^{2}, \quad \text { where } \\
& p(a, b):=a^{4} b^{2}+2 a^{3} b^{3}+a^{2} b^{4}+a^{4} b+5 a^{3} b^{2}+5 a^{2} b^{3}+a b^{4} \\
& +a^{4}-5 a^{3} b+21 a^{2} b^{2}-5 a b^{3}+b^{4}-2 a^{3}+5 a^{2} b+5 a b^{2}-2 b^{3}+a^{2}-a b+b^{2} \\
& x_{0}=(c, 1,1,1)^{\top}, \quad c>0, \quad\left\{\begin{array}{r}
x_{1} x_{2} x_{3} x_{4}=c \\
x_{1}-c x_{3}^{a} x_{4}^{b}=0 .
\end{array}\right.
\end{aligned}
$$

Proof. We follow the four cases of the table above: 
Case I): As in [5], the linear parts can be assumed in the following form:

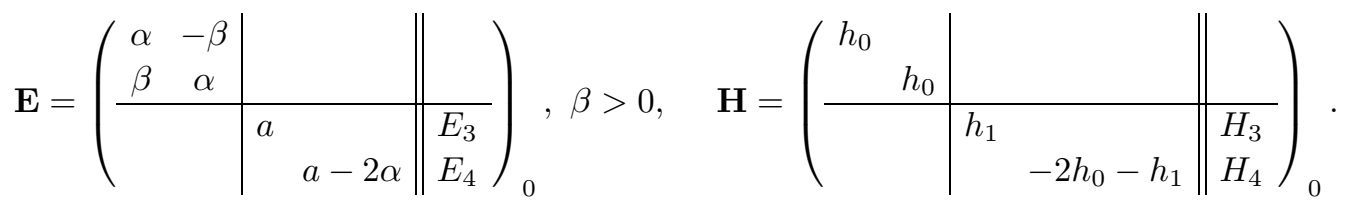

In addition we already assumed $E_{1}=E_{2}=H_{1}=H_{2}=0$ by T-design. All elements of the pencil of $E, H$ not proportional to $H$ have genuine complex as well as real eigenvalues. As to $h_{0}$ we have two subcases:

I.1) Subcase $h_{0} \neq 0$ : By subtracting a multiple of $H$ from $E$ and rescaling we can reach

$$
\mathbf{E}=\left(\begin{array}{ll|ll||l}
1 & & & \\
1 & & & \\
\hline & a & & E_{3} \\
E_{4}
\end{array}\right)_{0},
$$

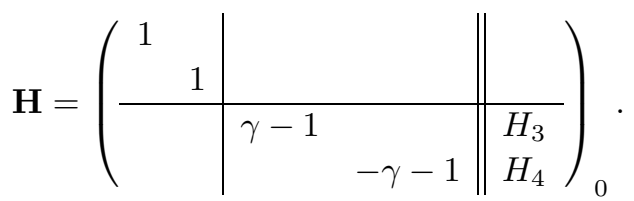

The ray of $E$, resp. $H$ is unique by having genuine pure imaginary eigenvalues, resp. real spectrum. We may assume

$$
\gamma \geq 0, \quad a \geq 0,
$$

the first by a possible interchange of the last two base vectors and the second by a possible sign change of $\mathbf{E}$ and interchange of the first two base vectors.

In the following subcases I.1.1), I.1.2), I.2.1) we write $b$ instead of $\gamma$.

I.1.1) Subcase: $a \neq 0$ : By TC-design, E, $\mathbf{H}$ come out as announced in $\|13\|$ with $\delta$ displayed there. The discussion of the zeros of $p$ runs as follows: $p(a, b)=0$ is equivalent to

$$
a^{4}+2 \lambda a^{2}+\mu=0
$$

where

$$
\lambda:=\frac{b^{4}+9 b^{2}-16}{b^{2}+32}, \quad \mu:=\frac{b^{2}\left(b^{2}-1\right)^{2}}{b^{2}+32}, \quad \omega:=\lambda^{2}-\mu=4 \cdot \frac{\left(b^{2}-4\right)^{2}\left(4-3 b^{2}\right)}{\left(b^{2}+32\right)^{2}} .
$$

Thus these zeros are described by

$$
a= \pm \sqrt{-\lambda \pm \sqrt{\omega}}
$$

There are real $a$ satisfying this iff $\omega \geq 0$ and $-\lambda+\sqrt{\omega} \geq 0$. (Observe $\omega \leq \lambda^{2}$, since $\mu \geq 0$.) In case $\lambda>0$ this is not possible, because then $\mu=0$, hence $b=0$ or $b=1$, which implies $\lambda<0$. In case $\lambda \leq 0$ this is fulfilled iff $b \leq \frac{2}{\sqrt{3}}$. So one has to require

$$
a>0, \quad b \geq 0, \quad a \neq \sqrt{-\lambda \pm \sqrt{\omega}} \text { if } b \leq \frac{2}{\sqrt{3}} .
$$

From the spectrum of $E, H$ follows that at most $E$ may be multiplied by -1 . However, checking the possibility of equations $\mathbf{T} \mathbf{E}^{\prime} \mathbf{T}^{-1}=\eta \mathbf{E}, \mathbf{T} \mathbf{H}^{\prime} \mathbf{T}^{-1}=\mathbf{H}$, where $\eta \in\{1,-1\}$ and $\mathbf{E}^{\prime}, \mathbf{H}^{\prime}$ are $\mathbf{E}, \mathbf{H}$ with $a$ replaced by $A$ and $b$ replaced by $B$, forces $\eta=1$ and $A=$ $a, B=b$. So the parameters $a, b$ are separating. 
I.1.2) Subcase: $a=0$ : Keeping $b \geq 0$ we have $-b-1<0$, so by TC-design $E_{4}=H_{4}=0$ and $(b-1) E_{3}=0$.

I.1.2.1) Subcase: $b \neq 1$ : Then in addition we may reach $E_{3}=H_{3}=0$, and the premetric requires $b>0$. So we obtain the normal forms in $\|14\|$. The remainder of the discussion is as in I.1.1), in particular $b$ is separating.

I.1.2.2) Subcase: $\gamma=1$ : Then the premetric shows $3 E_{3}^{2}-H_{3}^{2} \neq 0$. As above we may assume $E_{3} \geq 0$. Under the diagonal similarity $\mathbf{T}_{0}:=\operatorname{diag}\left(1,1, t_{3}, 1,1\right)$, solely $E_{3}, H_{3}$ are multiplied by $t_{3}$. So if $E_{3}>0$, we can achieve $E_{3}=1$, and if $E_{3}=0$ we can achieve $H_{3}=1$.

I.1.2.2.1) Subcase: $E_{3}>0$ : We have $H_{3} \geq 0, H_{3} \neq \sqrt{3}$ where the first condition can be reached by the additional diagonal similarity $\mathbf{T}_{0}^{\prime}:=\operatorname{diag}(1,-1,-1,1,1)$ and sign change of $\mathbf{E}$, which leaves $\mathbf{E}$ unaltered and changes in $\mathbf{H}$ the sign of $H_{3}$. Writing $H_{3}=: b$, we obtain the normal form $\|15\|$. The similarity test $\mathbf{T E T} \mathbf{T}^{-1}=\eta \mathbf{E}, \mathbf{T H}^{\prime} \mathbf{T}^{-1}=\mathbf{H}$ shows that $b$ is separating.

I.1.2.2.2) Subcase: $E_{3}=0$ : The normal form is as displayed in $\|16\|$.

I.2) Subcase: $h_{0}=0$ : The premetric requires $h_{1} \neq 0$ : So again we can reach $E_{3}=E_{4}=$ $H_{3}=H_{4}=0$ and have the same situation as in [5], end of E, case 1), leading to the normal forms in $\|17\|$. The ray of $E$, resp. $H$ is unique by having genuine complex eigenvalues and a double real one, resp. only real eigenvalues. Here, $\operatorname{rank}(H)=2$. Obviously, the parameter $a \geq 0$ is separating.

Case II): By T-design we can start with

$$
\mathbf{E}=\left(\begin{array}{cc|cc}
\alpha & -\beta & & \\
\beta & \alpha & & \\
\hline & \begin{array}{cc}
-\alpha_{1} & -\beta_{1} \\
\beta_{1} & -\alpha_{1}
\end{array}
\end{array}\right)_{0}, \beta>0, \beta_{1}>0, \quad \mathbf{H}=\left(\begin{array}{cc|cc||}
h & & \\
& h & & \\
\hline & & -h & \\
& & -h
\end{array}\right)_{0} .
$$

The premetric requires $h \neq 0$. As in [5], E case 2), by the usual operations we can immediately reach the normal forms $\|18\|$.

The sign condition for $a$ is obvious. If we have an $a>1$ then we can divide $E$ by $a$ and $H$ by -1 , interchange the two invariant planes and arrive at the above situation. All elements of the pencil not proportional to $H$ have only genuine complex eigenvalues. The spans of $E, H$ are unique by the property that the first has only pure imaginary eigenvalues and the second only real ones. From this one also sees that the parameter $a$ is separating.

Case III): Both translation parts can be made 0 and we have the same situation as in [5], E 3), immediately leading to the normal forms $\|19\|$ with $\delta$ displayed there.

The discussion of the zeros of the polynomial $p$ runs similar to the case I.1.1) above: If $32 b^{2}-1=0$ then $p(a, b)$ never vanishes. Otherwise, using the quantities

$$
\lambda:=\frac{16 b^{4}+9 b^{2}-1}{32 b^{2}-1}, \quad \mu:=-\frac{\left(b^{2}+1\right)^{2}}{32 b^{2}-1}, \quad \omega:=\lambda^{2}-\mu=4 \cdot \frac{b^{2}\left(4 b^{2}+3\right)\left(4 b^{2}+1\right)^{2}}{\left(32 b^{2}-1\right)^{2}},
$$


the vanishing of $p$ holds true iff

$$
a=\sqrt{-\lambda+\sqrt{\omega}}, \quad b>\frac{1}{\sqrt{32}} .
$$

So the final condition on $a, b$ is:

$$
0 \leq a \leq b, \quad a \neq \sqrt{-\lambda+\sqrt{\omega}} \text { if } b>\frac{1}{\sqrt{32}} .
$$

No element in the pencil of $E, H$ has a real spectrum. The spans of $E, H$ are unique by the property that their elements have at least one real eigenvalue; for $a=b$ however they are interchangeable. One also sees that the parameter pair $(a, b)$ is separating.

Case IV): Since $E, H$ can be assumed to be linearly independent we have the same forms for them as in [5], E (IV), and by TC-design both translation parts vanish, leading to the normal forms in $\|20\|$ with a rather complicated premetric expression, as displayed there. We were not able to establish a decomposition of the relevant polynomial $p(a, b)$ into squares. However, we can discuss the sign behaviour in a different way, using the finite group $\mathcal{P}$ introduced in [5], E case 4):

The group $\mathcal{P}$ is of order 24 and consists of projective maps acting in the $(a, b)$ parameter plane $\mathbf{R}^{2}$ such that two linear pencils are equivalent if their parameter pairs are equivalent under $\mathcal{P}$. There is a fundamental domain of $\mathcal{P}$, namely the triangle

$$
D:=\{(a, b) \mid 0 \leq a \leq b+1 \leq 1\},
$$

such that any point in $\mathbf{R}^{2}$ is equivalent to a point in $D$ under $\mathcal{P}$.

In order to find the zeros of $p$, it suffices to look for them in the domain with boundary $D$. The discussion of the minima of $p$ in $D$ is explicitly possible. It shows that $p$ is always nonnegative in $D$ with zeros only in $(0,0)$ and $(1,0)$. Besides the values $-2,-1,-\frac{1}{2}, 0,1$ for $a$ or $b$, no two elements of $D$ lead to similar pencils. This excludes the pairs $(0,-1)$ and $\left(0,-\frac{1}{2}\right)$ in $D$. But a direct inspection of the similarity relation for these two points shows that they also lead to non-similar pencils. Combining this with the exclusion of the seven points above this proves that every pencil is uniquely represented up to similarity by the parameter pairs in

$$
D^{\prime}:=D \backslash\{(0,0),(1,0)\}
$$

and that all nondegenerate orbits are hyperbolic.

The self-similarities can be cooked down in the following way: Assume similarity relations for $\mathbf{E}, \mathbf{H}$ :

$$
\begin{aligned}
& \mathbf{T E T}^{-1}=\alpha_{1} \mathbf{E}+\alpha_{2} \mathbf{H} \\
& \mathbf{T H T}^{-1}=\beta_{1} \mathbf{E}+\beta_{2} \mathbf{H}
\end{aligned}, \begin{aligned}
& T E T^{-1}=\alpha_{1} E+\alpha_{2} H \\
& T H T^{-1}=\beta_{1} E+\beta_{2} H
\end{aligned}, \operatorname{det} T \neq 0, \alpha_{1} \beta_{2}-\alpha_{2} \beta_{1} \neq 0,
$$

where we already gave the relations for the linear parts (which have the same coefficients).

If $E$ has four distinct eigenvalues then the same is true for $\alpha_{1} E+\alpha_{2} H$, and moreover $T$ is of the form $T=T_{0} S$ where $T_{0}$ is diagonal and $S$ a permutation matrix. The same form of $T$ results if $H$ has four distinct eigenvalues.

The eigenvalues of $E$, resp. $H$ are not distinct iff $a$, resp. $b$ has the values $-2,-1$, $-\frac{1}{2}, 0,1$. So, in $D^{\prime}$ this happens only for $a=0$, resp. $b=-1,-\frac{1}{2}, 0$. 
As to the translation part $t$ of $\mathbf{T}$, one deduces from the similarity relations and the fact that $\mathbf{E}, \mathbf{H}$ have vanishing translation parts that necessarily $T^{-1} t \in \operatorname{ker}(E) \cap \operatorname{ker}(H)$. For the above values of $a, b$ the kernels are as follows:

$$
\begin{aligned}
a=0: & \operatorname{ker}(E)=\operatorname{span}\left(e_{1}, e_{4}\right) \\
b=-1: & \operatorname{ker}(H)=\operatorname{span}\left(e_{2}, e_{3}\right) \\
b=-\frac{1}{2}: & \operatorname{ker}(H)=\operatorname{span}\left(e_{3}\right) \\
b=0: & \operatorname{ker}(H)=\operatorname{span}\left(e_{1}, e_{3}\right),
\end{aligned}
$$

otherwise $\operatorname{ker}(E)=\operatorname{span}\left(e_{4}\right)$ and $\operatorname{ker}(H)=\operatorname{span}\left(e_{3}\right)$. From this one reads off that $\operatorname{ker}(E) \cap$ $\operatorname{ker}(H)=\{0\}$ (hence $t=0$ ) and $T$ has the form specified above, unless both of $E, H$ have multiple eigenvalues.

This last case only happens for $(a, b)=(0,-1),\left(0,-\frac{1}{2}\right)$ (always in $\left.D^{\prime}\right)$. For these two parameter points, the similarity relations must be discussed in full detail, where one gets information on the coefficients ahead from the spectra. If $(a, b)=(0,-1)$, at most the values $0,1,-1$ are possible for $\alpha_{1}, \alpha_{2}, \beta_{1}, \beta_{2}$, and the calculation of $\mathbf{T}$, scanning through these coefficient possibilities, shows that it has no translation part and that its linear part is also of the above form $T_{0} S$. If $(a, b)=\left(0,-\frac{1}{2}\right)$ then, from the spectra, only $\left(\alpha_{1}, \alpha_{2}\right)=(1,0),(-1,0)$ and $\left(\beta_{1}, \beta_{2}\right)=(0,1),\left(-\frac{1}{2}, 1\right)$ come into consideration, and again the calculation of $\mathbf{T}$ leads to the same form.

In summary, for each $(a, b) \in D^{\prime}$ there are at least the self-similarities of the diagonal form $\mathbf{T}_{1}:=\operatorname{diag}\left(\tau_{1}, \tau_{2}, \tau_{3}, \tau_{4}, 1\right)$ and possibly some with vanishing translation part and linear part arising from $T_{1}$ by multiplication with a permutation matrix. This immediately shows that the initial points are classified by

$$
x_{1} x_{2} x_{3} x_{4}=c>0,
$$

so representative initial points are $(c, 1,1,1)^{\top}$ with $c>0$.

5. The noncommutative case. Finally we have to treat the noncommutative Lie algebras with generators $\mathbf{E}, \mathbf{H}$, satisfying $[\mathbf{E}, \mathbf{H}]=\mathbf{E}$. Such a pencil can never be of dimension 1, and by this relation the ray of $\mathbf{E}$ is unique in the pencil and consists of nilpotent elements. The same is true for the corresponding linear pencil because $[E, H]=$ $E$; see [5], Sect. 5 for details. So it is reasonable to go through all Jordan possibilities for $E$, and to supplement it suitably as described in Sect. 2 in order to obtain normal forms for $\mathbf{E}, \mathbf{H}$. A special feature here is that the orbits belonging to the same group may split into many different congruence classes.

C. Theorem. Let $\Gamma^{\bullet}$ be a two-dimensional noncommutative Lie subalgebra of $\mathbf{S A}(4, \mathbf{R})^{\bullet}$, whose orbits are not all degenerate with respect to the Burstin-Mayer metric.

Then $\Gamma^{\bullet}$ is similar to one of the following fifteen cases $\|21\|, \ldots,\|35\|$, specifying $\Gamma^{\bullet}$ by the given generators $\mathbf{E}, \mathbf{H}$, the initial points $x_{0}$, and the discriminants $\delta$ and implicit equations as in Theorem $A$. For the group families $\|23\|,\|24\|,\|29\|$ there are several distinct orbit families.

Within each case, the parameters possibly occurring in $\mathbf{E}, \mathbf{H}$, resp. $x_{0}$ are separating under the given conditions between similarity classes of pencils, resp. congruence classes 
of orbits; however, all $\mathbf{H}$, resp. $H$ in the pencil satisfying $[\mathbf{E}, \mathbf{H}]=\mathbf{E}$, resp. $[E, H]=E$ are equally entitled, in particular all the $H$ have the same Jordan normal form.

(I) $E$ is nilpotent of degree 4 :

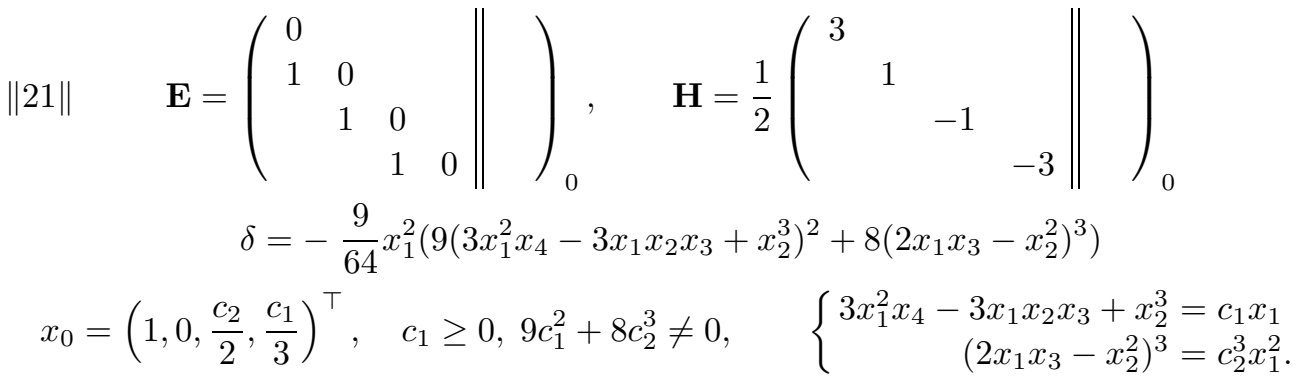

(II.1) $E$ is nilpotent of degree 3 :

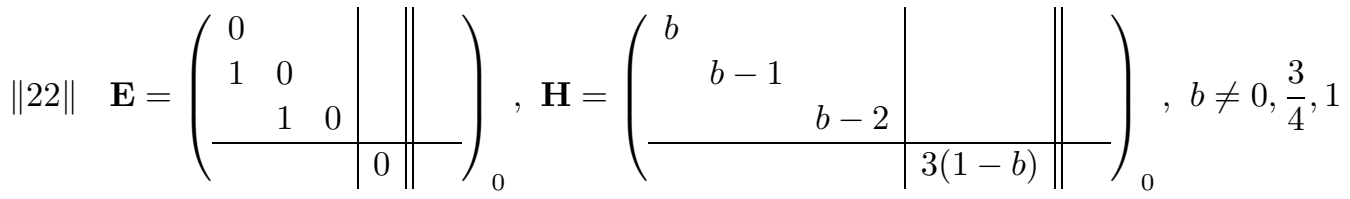

$$
\begin{gathered}
\delta=-\frac{9}{4} b^{2}(4 b-3)^{2}(b-1)^{2} x_{1}^{6} x_{4}^{2} \\
x_{0}=(1,0, c, 1)^{\top}, \quad c \geq 0, \quad\left\{\begin{aligned}
\left(2 x_{1} x_{3}-x_{2}^{2}\right)^{3} x_{4}^{2} & =8 c^{3} \\
x_{4}-x_{1}^{3 \frac{1-b}{b}} & =0 .
\end{aligned}\right.
\end{gathered}
$$

(II.2) $E$ is nilpotent of degree 3 :

||23\|

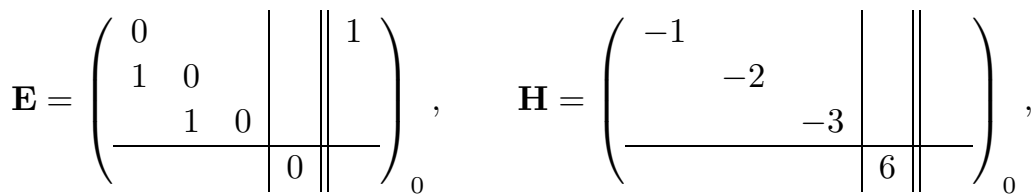

$$
\begin{aligned}
& \delta=-9 x_{4}^{2}\left(81\left(x_{1}^{3}-3 x_{1} x_{2}+3 x_{3}\right)^{2}-32\left(x_{1}^{2}-2 x_{2}\right)^{3}\right) \\
& \left\{\begin{array}{l}
x_{0}=\left(0,-\frac{\varepsilon}{2}, \frac{c_{1}}{3}, c_{2}\right)^{\top} \\
\varepsilon \in\{1,-1\}, c_{1}>0, c_{2}>0
\end{array}, \quad\left\{\begin{array}{c}
\left(x_{1}^{3}-3 x_{1} x_{2}+3 x_{3}\right)^{2}=\varepsilon c_{1}^{2}\left(x_{1}^{2}-2 x_{2}\right)^{3} \\
\left(x_{1}^{2}-2 x_{2}\right)^{3} x_{4}=\varepsilon c_{2}^{2} .
\end{array}\right.\right.
\end{aligned}
$$

||23.a\|

$$
\begin{gathered}
x_{0}=\left(0,-\frac{\varepsilon}{2}, 0, c_{2}\right)^{\top}, \quad \varepsilon \in\{1,-1\}, c_{2}>0, \quad\left\{\begin{aligned}
x_{1}^{3}-3 x_{1} x_{2}+3 x_{3} & =0 \\
\left(x_{1}^{2}-2 x_{2}\right)^{3} x_{4} & =\varepsilon c_{2} .
\end{aligned}\right. \\
x_{0}=\left(0,0,1, c_{2}\right)^{\top}, \quad c_{2}>0, \quad\left\{\begin{aligned}
\left(x_{1}^{3}-3 x_{1} x_{2}+3 x_{3}\right)^{2} x_{4} & =9 c_{2} \\
x_{1}^{2}-2 x_{2} & =0 .
\end{aligned}\right.
\end{gathered}
$$

(II.3) $E$ is nilpotent of degree 3 :

||24\|

$$
\mathbf{E}=\left(\begin{array}{ccc|c||c}
0 & & & & \\
1 & 0 & & & \\
& 1 & 0 & & \\
\hline & & & 0 & 1
\end{array}\right),
$$

$$
\mathbf{H}=\frac{1}{3}\left(\begin{array}{lll|l||l}
4 & & & & \\
& 1 & & & \\
& & -2 & & \\
\hline & & & -3 &
\end{array}\right)_{0}
$$


$\|24 . \mathrm{a}\| \quad\left\{\begin{array}{c}x_{0}=\left(1,0, \frac{1}{2} c_{1}^{2}\left(c_{2}-1\right), c_{1}\right)^{\top} \\ c_{1}>0, c_{2} \neq-\frac{19}{128}\end{array},\left\{\begin{array}{c}\left(x_{1} x_{4}-x_{2}\right)^{4}=c_{1}^{4} x_{1} \\ x_{1}\left(x_{1} x_{4}^{2}-2 x_{2} x_{4}+2 x_{3}\right)=c_{2}\left(x_{1} x_{4}-x_{2}\right)^{2} .\end{array}\right.\right.$

$$
x_{0}=(1,0, c, 0)^{\top}, \quad c \neq 0 \quad\left\{\begin{array}{rl}
x_{1} x_{4}-x_{2} & =0 \\
x_{1}\left(x_{1} x_{4}^{2}-2 x_{3}\right)^{2} & =4 c^{2}
\end{array} .\right.
$$

(II.4) E is nilpotent of degree 3:

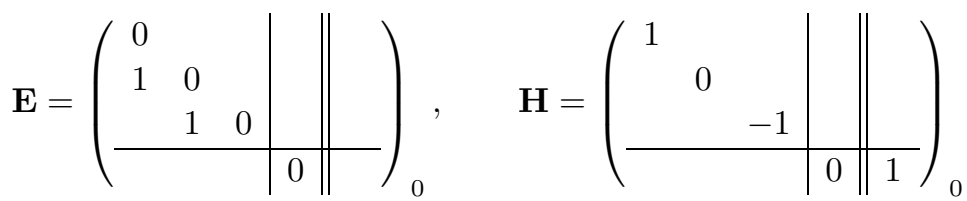

$$
\begin{aligned}
& \delta=-\frac{1}{4} x_{1}^{6} \\
& x_{0}=(1,0, c, 0)^{\top}, \quad c \in \mathbf{R}, \quad\left\{\begin{aligned}
2 x_{1} x_{3}-x_{2}^{2} & =2 c \\
\ln x_{1}-x_{4} & =0 .
\end{aligned}\right.
\end{aligned}
$$

(II.5) E is nilpotent of degree 3 :

$\|26\|$

$$
\begin{aligned}
& \mathbf{E}=\left(\begin{array}{ccc|c||c}
0 & & & & \\
1 & 0 & & & \\
& 1 & 0 & & \\
\hline & & 0 &
\end{array}\right)_{0}, \\
& \mathbf{H}=\left(\begin{array}{lll|l||l}
2 & & & & \\
& 1 & & & \\
& & 0 & & 1 \\
\hline & & & -3 &
\end{array}\right)_{0} \\
& \delta=-225 x_{1}^{6} x_{4}^{2} \\
& x_{0}=(c, 0,0,1)^{\top}, \quad c \neq 0, \quad\left\{\begin{aligned}
x_{1}^{3} x_{4}^{2} & =c^{3} \\
6 x_{1} x_{3}-3 x_{2}^{2}+2 x_{1} \ln x_{4} & =0 .
\end{aligned}\right.
\end{aligned}
$$

(II.6) E is nilpotent of degree 3:

$\|27\|$

$$
\begin{aligned}
& \mathbf{E}=\left(\begin{array}{ccc|c||c}
0 & & & & \\
1 & 0 & & & \\
& 1 & 0 & & \\
\hline & & 0 &
\end{array}\right)_{0} \\
& \mathbf{H}=\frac{1}{4}\left(\begin{array}{ccc|c||c}
5 & & & & \\
& 1 & & & \\
& & -3 & 4 & \\
\hline & & -3 &
\end{array}\right)_{0} \\
& \delta=-\frac{225}{256} x_{1}^{6} x_{4}^{2} \\
& x_{0}=(1,0,0, c)^{\top}, \quad c \neq 0, \quad\left\{\begin{aligned}
x_{1}^{3} x_{4}^{5} & =c^{5} \\
5\left(2 x_{1} x_{3}-x_{2}^{2}\right)-8 c x_{1}^{2 / 5} \ln x_{1} & =0 .
\end{aligned}\right.
\end{aligned}
$$

(II.7) E is nilpotent of degree 3:

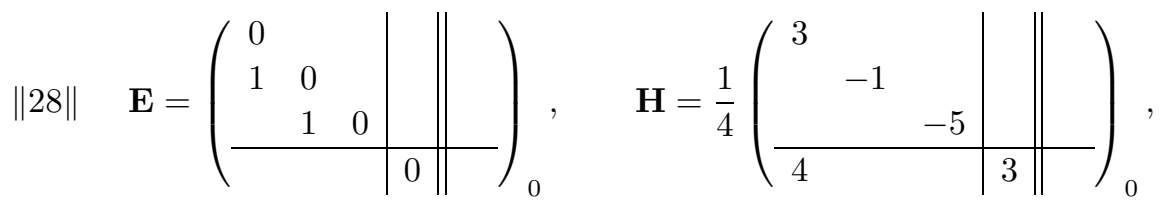




$$
\begin{gathered}
\delta=-\frac{81}{1024} x_{1}^{8} \\
x_{0}=(1,0, c, 0)^{\top}, \quad c \in \mathbf{R}, \quad\left\{\begin{array}{c}
x_{1}^{2}\left(2 x_{1} x_{3}-x_{2}^{2}\right)^{3}=8 c^{3} \in \mathbf{R} \\
3 x_{4}-4 x_{1} \ln x_{1}=0 .
\end{array}\right.
\end{gathered}
$$

(III) $E$ is nilpotent of degree 2 and of rank 2 :

$\|29\|$

$$
\begin{aligned}
& \mathbf{E}=\left(\begin{array}{ll|ll||l}
0 & & & & \\
1 & 0 & & & \\
\hline & & 0 & & 1 \\
& & 1 & 0 &
\end{array}\right)_{0}
\end{aligned}
$$

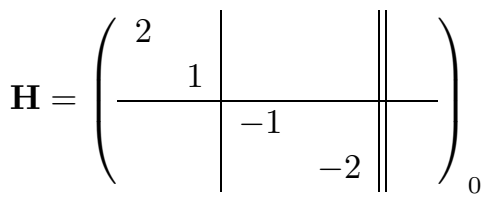

$$
\begin{aligned}
& \delta=x_{1}^{2}\left(64 x_{1}^{2}\left(x_{3}^{2}-2 x_{4}\right)-\left(x_{1} x_{3}-x_{2}\right)^{2}\right)
\end{aligned}
$$

||29.a\|

$$
\left\{\begin{array}{l}
x_{0}=\left(c_{1},-1,0,-c_{2}\right)^{\top} \\
c_{1} c_{2}>0, c_{1}^{2} c_{2} \neq \frac{1}{128}
\end{array}, \quad\left\{\begin{aligned}
x_{1}^{2}\left(x_{3}^{2}-2 x_{4}\right)^{2} & =4 c_{1}^{2} c_{2}^{2} \\
\left(x_{1} x_{3}-x_{2}\right)^{2}\left(x_{3}^{2}-2 x_{4}\right) & =2 c_{2} .
\end{aligned}\right.\right.
$$

\|29.b\|

$$
x_{0}=(1, c, 0,0)^{\top}, \quad c>0, \quad\left\{\begin{array}{c}
x_{3}^{2}-2 x_{4}=0 \\
\left(x_{1} x_{3}-x_{2}\right)^{2}=c^{2} x_{1} .
\end{array}\right.
$$

\|29.c $\|$

$$
x_{0}=(1,0,0,-c)^{\top}, \quad c \neq 0, \quad\left\{\begin{array}{c}
x_{1} x_{3}-x_{2}=0 \\
x_{1}\left(x_{3}^{2}-2 x_{4}\right)=2 c .
\end{array}\right.
$$

(IV.1) E is nilpotent of degree 2 and of rank 1 :

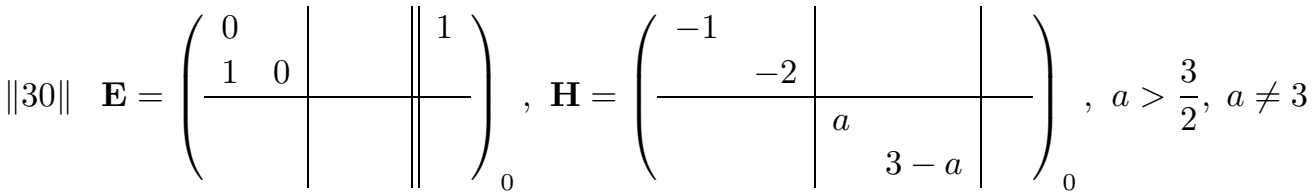

$$
\begin{aligned}
& \delta=-\frac{1}{4} a^{2}(a-3)^{2}(2 a-3)^{2} x_{3}^{2} x_{4}^{2} \\
& x_{0}=(0,-c, 1,1)^{\top}, \quad c \in \mathbf{R}, \quad\left\{\begin{aligned}
x_{3}^{2} x_{4}^{2}\left(x_{1}^{2}-2 x_{2}\right)^{3} & =8 c^{3} \\
x_{4} & =x_{3}^{\frac{3-a}{a}} .
\end{aligned}\right.
\end{aligned}
$$

If $a=5$ then only $c=0$ must be considered.

(IV.2) $E$ is nilpotent of degree 2 and of rank 1 :

$\|31\|$

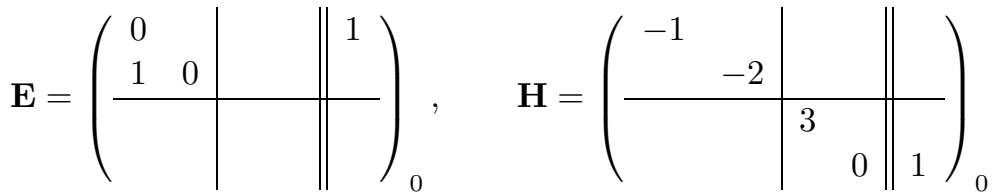

$$
\begin{aligned}
& \delta=-\frac{81}{4} x_{3}^{2} \\
& x_{0}=(0,-c, 1,0)^{\top}, \quad c \in \mathbf{R}, \quad\left\{\begin{aligned}
x_{3}^{2}\left(x_{1}^{2}-2 x_{2}\right)^{3} & =8 c^{3} \\
3 x_{4} & =\ln x_{3} .
\end{aligned}\right.
\end{aligned}
$$

(IV.3) $E$ is nilpotent of degree 2 and of rank 1 :

$\|32\|$

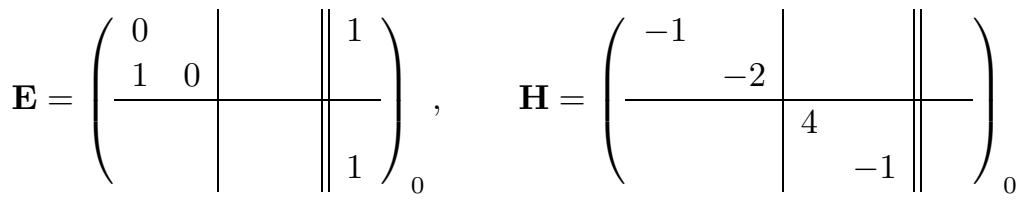




$$
\begin{gathered}
\delta=-100\left(x_{1}-x_{4}\right)^{2} x_{3}^{2} \\
x_{0}=(0,-c, 1,1)^{\top}, \quad c \in \mathbf{R}, \quad\left\{\begin{array}{c}
\left(x_{1}^{2}-2 x_{2}\right)^{2} x_{3}=4 c^{2} \\
\left(x_{1}-x_{4}\right)^{4} x_{3}=1 .
\end{array}\right.
\end{gathered}
$$

(IV.4) E is nilpotent of degree 2 and of rank 1 :

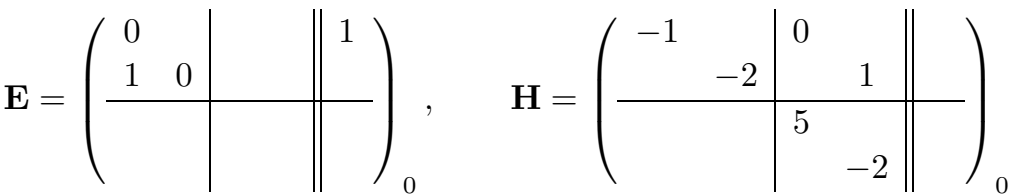

$$
\begin{aligned}
& \delta=-1225 x_{3}^{2} x_{4}^{2} \\
& x_{0}=(0,0,1, c)^{\top}, \quad c \neq 0, \quad\left\{\begin{aligned}
x_{3}^{2} x_{4}^{5} & =c^{5} \\
5 x_{1}^{2}-10 x_{2}+2 x_{4} \ln x_{3} & =0 .
\end{aligned}\right.
\end{aligned}
$$

(IV.5) E is nilpotent of degree 2 and of rank 1 :

$\|34\|$

$$
\begin{aligned}
& \mathbf{E}=\left(\begin{array}{ll|l||l}
0 & & & 1 \\
1 & 0 & & \\
\hline & & &
\end{array}\right)_{0}, \\
& \mathbf{H}=\frac{1}{2}\left(\begin{array}{ll|l||l}
-2 & & & \\
& -4 & & \\
& & 3 & \\
& 2 & 3
\end{array}\right)_{0} \\
& \delta=-\frac{81}{64} x_{3}^{4} \\
& x_{0}=(0,-c, 1,0)^{\top}, \quad c \in \mathbf{R}, \quad\left\{\begin{aligned}
\left(x_{1}^{2}-2 x_{2}\right)^{3} x_{3}^{4} & =8 c^{3} \\
3 x_{4} & =2 x_{3} \ln x_{3} .
\end{aligned}\right.
\end{aligned}
$$

(IV.6) E is nilpotent of degree 2 and of rank 1 :

$$
\begin{gathered}
\mathbf{E}=\left(\begin{array}{ll||l}
0 & & \\
1 & 0
\end{array}\right. \\
\hline
\end{gathered}
$$

Proof. We have to go through all possibilities for $E$ to be nilpotent. The calculation of the self-similarities from $\mathbf{T E T}{ }^{-1}=\alpha_{1} \mathbf{E}$, THT $^{-1}=\beta_{1} \mathbf{E}+\beta_{2} \mathbf{H}$ will show that in each case the values $\alpha_{1}=\beta_{2}=1$ and arbitrary $\beta_{1}$ are possible. Thus, for any $\beta_{1}, \mathbf{H}$ and $\mathbf{H}^{\prime}:=\mathbf{H}+\beta_{1} \mathbf{E}$ are similar with a $\mathbf{T}$, leaving $\mathbf{E}$ fixed. In particular, $\mathbf{H}, \mathbf{H}^{\prime}$ have the same Jordan normal form (and analogously for $H, H^{\prime}$ ).

Case I): $E$ is nilpotent of degree 4: With respect to $E, H$, we have the same situation as in [5], F (I). Since $H$ is regular, the linear part of $\mathbf{H}$ can be made 0 , and then $[\mathbf{E}, \mathbf{H}]=\mathbf{E}$ implies that the linear part of $\mathbf{E}$ vanishes, too. So immediately we arrive at the normal forms $\|21\|$ and the initial point conditions there. 
Case II): $E$ is nilpotent of degree 3: There are several cases for the linear parts $E, H$, as described in [5], F (II). If in the notation there $\alpha_{1} \neq \frac{3}{4}, \frac{5}{4}$ then $E$ may be assumed in Jordan normal form and $H$ as $\operatorname{diag}(b, b-1, b-2,3(1-b))$ with $b \neq \frac{3}{4}, \frac{5}{4}$.

II.1) Subcase: $b \neq \frac{3}{4}, \frac{5}{4}:$

II.1.1) Subcase: $b \neq 0,1,2,-1, \frac{4}{3}$ : By exclusion of the first three values we obtain a nonsingular $H$, and by exclusion of the last two values both translation parts can be made zero by TC-design. This immediately gives the normal forms $\|22\|$ (disregarding the conditions on $b$ there for the moment). As in [5], we see that $b$ separates between similarity classes of the possible pencils of $E, H$ (and a fortiori between those for $\mathbf{E}, \mathbf{H}$ ).

II.1.2) Subcase: $b=-1$ : Then $H$ is still regular, and by TC-design we can assume $E_{i}=0$ for $i \neq 1$ and $H_{i}=0$ for all $i$. We may assume $E_{1} \neq 0$ because otherwise we can apply subcase II.1.1). So $b=-1$ must be adjoined to $\|22\|$ ( $b$ being still separating there). Here, by H-design, we can achieve $E_{1}=1$, thus arriving at $\|23\|$ with $\delta$ displayed there.

In contrast to the former situations, the initial point discussion splits into three different cases, according to the zero behaviour of the two quantities

$$
X:=x_{1}^{3}-3 x_{1} x_{2}+3 x_{3}, \quad \xi:=x_{1}^{2}-2 x_{2} .
$$

If $X \neq 0, \xi \neq 0$ the equivalence classes for initial points are parametrized by triplets $\left(\varepsilon, c_{1}, c_{2}\right)$ with

$$
\varepsilon \in\{1,-1\}, \quad c_{1}>0, \quad \varepsilon c_{1}^{2} \neq \frac{32}{81}, \quad c_{2}>0
$$

such that

$$
\varepsilon=\operatorname{sign}(\xi), \quad X^{2}=\varepsilon c_{1}^{2} \xi^{3}, \quad \xi^{6} x_{4}^{2}=c_{2}^{2} .
$$

Representative initial points are then as displayed in $\|23 . \mathrm{a}\|$.

If $X=0, \xi \neq 0$ the equivalence classes for initial points are parametrized by pairs $\left(\varepsilon, c_{2}\right)$ with $\varepsilon \in\{1,-1\}, c_{2}>0$ such that

$$
\operatorname{sign}(x)=\varepsilon, \quad X=0, \quad \xi^{6} x_{4}^{2}=c_{2}^{2},
$$

with representative initial points as displayed in $\|23 . \mathrm{b}\|$.

If $X \neq 0, \xi=0$ the equivalence classes of orbits are classified by $c_{2}>0$ such that

$$
\xi=0, \quad X^{4} x_{4}^{2}=81 c_{2}^{2},
$$

with representative initial points as displayed in $\|23 . c\|$.

II.1.3) Subcase: $b=\frac{4}{3}:$ As in II.1.2) the translation parts can be arranged such that at most $E_{4}$ doesn't vanish. So we may assume $E_{4} \neq 0$, in fact as above $E_{4}=1$. The case $E_{4}=0$ must be adjoined to $\|22\|$ ( $b$ is then still separating there). Here we arrive at $\|24\|$ with $\delta$ displayed there. Also in this case the orbit discussion falls into two parts, according to $x_{1} x_{4}-x_{2} \neq 0,=0$, the result being displayed in $\| 24$.a $\|\|$,24 .b $\|$.

II.1.4) Subcase: $b=0$ : By TC-design both translation parts vanish. Then the premetric is 0 , so this case doesn't exist here. This is also clear from [5], E case (II), stating that all orbits are contained in hyperplanes. 
II.1.5) Subcase: $b=1$ : By TC-design we can reach that all $E_{i}, H_{i}$ vanish, except possibly $H_{4}$, but then $H_{4} \neq 0$ by the premetric. By rescaling $e_{4}$, we achieve $H_{4}=1$, thus arriving at $\|25\|$.

II.1.6) Subcase: $b=2$ : By TC-design again all $E_{i}, H_{i}$ vanish, except possibly $H_{3}$. If $H_{3}=0$, we are back in the case II.1.1), so must adjoin this value to $\|22\|$, without altering the separation property of $b$ there. So here we may assume $H_{3} \neq 0$, in fact $H_{3}=1$ by rescaling $e_{3}$, and arrive at $\|26\|$.

II.2) Subcase: $b=\frac{5}{4}$ : This is the case $\alpha_{1}=\frac{5}{4}$ in the notation of [5], F (II). By TC-design both translation parts vanish. Thus, as in the linear case of [5], the $(3,4)$-entry of $H$ is any real. If this is 0 we are back in the case II.1.1), adjoining this value to $\|22\|$ without destroying the separation property of $b$. Here we are left with a nonzero value of this entry, in fact can make it 1 by rescaling $e_{4}$. Thus completely as in [5] we reach $\|27\|$.

II.3) Subcase: $b=\frac{3}{4}$ : This is the case $\alpha_{1}=\frac{3}{4}$ in the notation of [5], F (II). By TC-design again both translation parts are zero Thus, as in the linear situation, the $(4,3)$-entry of $H$ is any real, where the premetric demands a value $\neq 0$. In fact, it can be made 1 by rescaling $e_{4}$. Thus completely as in [5] we reach $\|28\|$.

Case III): $E$ is nilpotent of degree 2 and of rank 2: There are several cases for the linear parts $E, H$, as described in [5], F (III):

III.1) Subcase: there is a diagonalizable element $H$ :

III.1.1) Subcase: all eigenvalues of $H$ are real: Then we saw there that simultaneously $E$ can be assumed in Jordan normal form and $H$ as $\frac{1}{2} \operatorname{diag}(b+1, b-1,-b+1,-b-1)$ with $b \geq 0$.

If $b \neq 1$ the translation part of $\mathbf{H}$ can be made 0 . If in addition $b \neq 3$ the commutator relation forces the translation part of $\mathbf{H}$ to vanish. Then the premetric degenerates. If $b=1$ then by TC-design all $E_{i}, H_{i}$ vanish, except possibly $H_{2}$. But then again the premetric vanishes.

So we are left with $b=3$. In this case by TC-design all $H_{i}$ vanish and also all $E_{i}$ except possibly $E_{3}$, but the premetric requires indeed $E_{3} \neq 0$. Then by H-design we can achieve $E_{3}=1$, keeping $H$, so we reach $\|29\|$ with $\delta$ displayed there. In this case the discussion of initial points again splits into three cases $\| 29$.a - c $\|$ according to the conditions $x_{1} x_{3}-x_{2} \neq 0,=0, x_{3}^{2}-2 x_{4} \neq 0,=0$.

III.1.2) Subcase: $H$ has a genuine complex eigenvalue: The linear parts can be brought to normal forms as in [5], F (III.2). Then by TC-design both translation parts vanish, and the premetric turns out to be degenerate.

III.2) Subcase: there is no diagonalizable element $H$ : Using [5], Lemma 5.2 we can achieve

$$
\mathbf{E}=\left(\begin{array}{cc|cc||c|c||c}
0 & & & & E_{1} \\
1 & 0 & & & E_{2} \\
\hline & 0 & & E_{3} \\
& 1 & 0 & E_{4}
\end{array}\right), \quad \mathbf{H}=\left(\begin{array}{cccc|c}
a & & \alpha_{2} & & H_{1} \\
& a-1 & \beta_{2} & \alpha_{2} & H_{2} \\
\hline \alpha_{1} & & b & & H_{3} \\
\beta_{1} & \alpha_{1} & & b-1 & H_{4}
\end{array}\right), \quad b:=1-a,
$$

and by symmetry assume $a \geq b$, i.e. $a \geq \frac{1}{2}$. 
By T-design we can reach $E_{2}=E_{4}=H_{4}=0$. The characteristic polynomial of $H$ is

$$
\chi_{H}(\xi)=\left(\xi^{2}-\xi+a b-\alpha_{1} \alpha_{2}\right)\left(\xi^{2}+\xi+a b-\alpha_{1} \alpha_{2}\right) .
$$

Since $H$ is not diagonalizable, $\chi_{H}$ must have at least one zero of multiplicity $\geq 2$. If the constant term in the given factorization of $\chi_{H}$ is $\neq 0$ this is only possible if this term has the value $\frac{1}{4}$. So necessarily

$$
a b-\alpha_{1} \alpha_{2} \in\left\{0, \frac{1}{4}\right\} .
$$

The commutator relation is equivalent to the four equations

$$
\begin{aligned}
& (a+1) E_{1}+\alpha_{2} E_{3}=0 \quad H_{1}=\beta_{2} E_{3} \\
& \alpha_{1} E_{1}+(b+1) E_{3}=0, \quad H_{3}=\beta_{1} E_{1} .
\end{aligned}
$$

In both cases of (5.1) this implies $E_{1}=E_{3}=H_{1}=H_{3}=0$, and then the premetric turns out to be degenerate. So this case doesn't exist either.

Case IV): $E$ is nilpotent of degree 2 and of rank 1: This case has no counterpart in [5] because the centro-affine metric vanishes for its orbits.

We have $E=J(2,0)+J(1,0)+J(1,0)$ in Jordan normal form. By T-design of $\mathbf{E}$, we can reach $E_{2}=0$. Calculating $\exp (u \mathbf{E})$ we observe that the $u$-lines become straight unless $E_{1} \neq 0$. Since ruled surfaces cannot have a regular premetric, we must have $E_{1} \neq 0$, and in fact can reach $E_{1}=1$ by H-design. $\mathbf{E}$ is then nilpotent of degree 3 .

The most general $\mathbf{H}$ satisfying $[\mathbf{E}, \mathbf{H}]=\mathbf{E}$ is of the form

$$
\left(\begin{array}{cc|cc||c}
-1 & & 0 & 0 & H_{1} \\
\tau_{10} & -2 & \tau_{9} & \tau_{8} & \tau_{7} \\
\hline f_{1} & 0 & \tau_{6} & \tau_{5} & \tau_{4} \\
f_{2} & 0 & \tau_{3} & \tau_{2} & \tau_{1}
\end{array}\right)_{0}
$$

where the $\tau_{i}$ are free and $H_{1}, f_{1}, f_{2}$ are determined by them (without $\tau_{1}, \tau_{4}, \tau_{7}$ ) and by $E_{3}, E_{4}$. Subtracting a multiple of $\mathbf{E}$ we can assume $\tau_{10}=0$.

For further reduction we study first the possible linear parts, i.e.

$$
E=\left(\begin{array}{cc|c}
0 & & \\
1 & 0 & \\
\hline &
\end{array}\right), \quad H=\left(\begin{array}{cc|cc}
-1 & & 0 & 0 \\
& -2 & q_{1} & q_{2} \\
\hline f_{1} & 0 & h_{11} & h_{12} \\
f_{2} & 0 & h_{21} & h_{22}
\end{array}\right), \quad \operatorname{trace}(H)=0 .
$$

The most general base change leaving the Jordan normal form $E$ fixed is described by

$$
T=\left(\begin{array}{cc|cc}
\tau_{9} & & 0 & 0 \\
\tau_{10} & \tau_{9} & \tau_{8} & \tau_{7} \\
\hline \tau_{6} & 0 & \tau_{5} & \tau_{4} \\
\tau_{3} & 0 & \tau_{2} & \tau_{1}
\end{array}\right), \quad \operatorname{det}(T)=\tau_{9}^{2}\left(\tau_{5} \tau_{1}-\tau_{4} \tau_{2}\right) \neq 0
$$

with otherwise free (new) $\tau_{1}, \ldots, \tau_{10}$.

Choosing $T_{0}$ as $T$ with $\tau_{9}=1, \tau_{10}=\tau_{8}=\tau_{7}=\tau_{6}=\tau_{3}=0$, these $T_{0}$ don't change the specific form of $H$, and the lower-right blocks of $T_{0}$ operate on that of $H$ as general $(2 \times 2)$-similarities. So hereby we can give the lower-right block of $H$ one of the three real 
Jordan normal forms $\operatorname{diag}(a, b), \mathcal{J}_{1}(2), \mathcal{K}_{1}(2)$. The fine distinction follows these three possibilities:

IV.1) Subcase: $\left(\begin{array}{ll}h_{11} & h_{12} \\ h_{21} & h_{22}\end{array}\right)=\left(\begin{array}{ll}a & \\ & b\end{array}\right)$ : By the interchange of $e_{3}, e_{4}$ we may assume $a \geq b$, i.e. $a \geq \frac{3}{2}$, and then $a$ is separating for these pencils, as follows from the spectrum of the $H$.

Choosing $T_{1}$ as $T$ with $\tau_{9}=\tau_{5}=\tau_{1}=1, \tau_{10}=\tau_{4}=\tau_{2}=0$ the form of $H$ is kept, however with possibly nonvanishing $(2,1)$-entry and new $f_{i}, q_{i}$, namely

$$
\begin{array}{ll}
\bar{f}_{1}=f_{1}-(a+1) \tau_{6} & \bar{q}_{1}=q_{1}+(a+2) \tau_{8} \\
\bar{f}_{2}=f_{2}-(b+1) \tau_{3} & \bar{q}_{2}=q_{2}+(b+2) \tau_{7} .
\end{array}
$$

IV.1.1) Subcase: $a \neq 4,5$ : Under this assumption the system (5.2) shows that the $f_{i^{-}}$ and $q_{i}$-entries of $H$ can be made 0 , and also its $(2,1)$-entry by subtracting a multiple of $E$ :

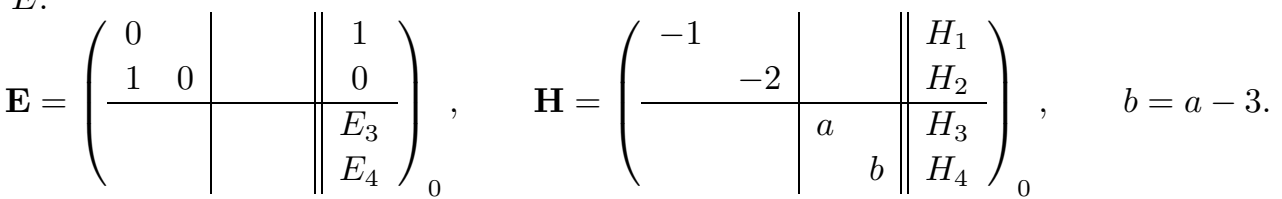

By T-design we can achieve $H_{2}=H_{3}=0$ (and, if $b \neq 0$, also $H_{4}=0$ ), and the commutator relation then yields $E_{3}=E_{4}=H_{1}=0$. Moreover, the premetric requires $a \neq b$, i.e. $a \neq \frac{3}{2}$. If $b=0$, the premetric requires $H_{4} \neq 0$, and we can additionally achieve $H_{4}=1$ by rescaling $e_{4}$. So we have two subcases here:

IV.1.1.1) Subcase: $a>\frac{3}{2}, a \neq 3$ : Here we arrive at $\|30\|$.

IV.1.1.2) Subcase: $a=3$ : Here we arrive at $\|31\|$.

IV.1.2) Subcase: $a=4$ : Here the equations (5.2) can only be used to achieve $\bar{f}_{1}=$ $\bar{q}_{1}=\bar{q}_{2}=0$, so

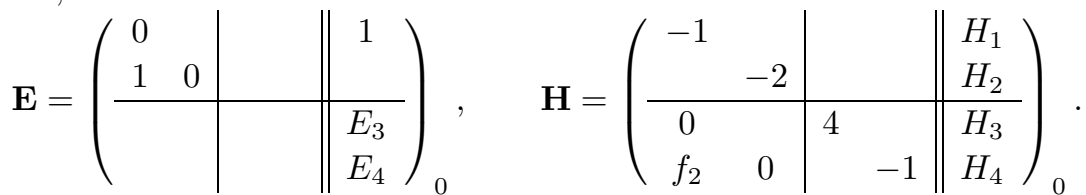

By TC-design we can reach that all $H_{i}=0$ and $f_{2}=E_{3}=0$, moreover, if $E_{4} \neq 0$, by rescaling $e_{4}: E_{4}=1$. If $E_{4}=0$ then we can adjoin this to the case IV.1.1.1), so are left here with $E_{4}=1$, leading to $\|32\|$.

IV.1.3) Subcase: $a=5$ : Here the equations (5.2) can only be used to achieve $\bar{f}_{1}=$ $\bar{f}_{2}=\bar{q}_{1}=0$, so

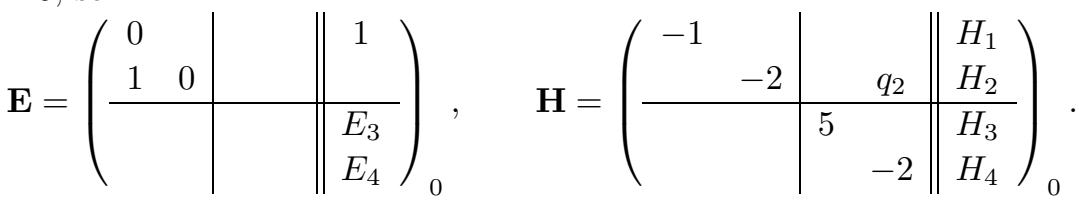

By TC-design we can reach that all $H_{i}=0$ and $E_{3}=E_{4}=0$, moreover, if $q_{2} \neq 0$, by rescaling $e_{4}: q_{2}=1$. If $q_{2}=0$ then we can adjoin this to the case IV.1.1.1), so are left here with $q_{2}=1$, leading to $\|33\|$. 
IV.2) Subcase: $\left(\begin{array}{ll}h_{11} & h_{12} \\ h_{21} & h_{22}\end{array}\right)=\left(\begin{array}{cc}\frac{3}{2} & \\ 1 & \frac{3}{2}\end{array}\right)$ : Choosing $T_{1}$ as above, the form of $H$ is kept, however with possibly nonvanishing $(2,1)$-entry and new $f_{i}, q_{i}$, namely

$$
\begin{array}{lll}
\bar{f}_{1}=f_{1}-\frac{5}{6} \tau_{6} & \bar{q}_{1}=q_{1}+\frac{7}{2} \tau_{8}+\tau_{7} \\
\bar{f}_{2}=f_{2}-\frac{5}{2} \tau_{3}-\tau_{6} & \bar{q}_{2}=q_{2}+\frac{7}{2} \tau_{7} .
\end{array}
$$

So the $f_{i^{-}}$and $q_{i}$-entries of $H$ can be made 0 , and also its $(2,1)$-entry by subtracting a multiple of $E$. As to the translation parts we can achieve by TC-design that all $H_{i}=0$ and $E_{3}=E_{4}=0$, arriving at $\|34\|$.

IV.3) Subcase: $\left(\begin{array}{ll}h_{11} & h_{12} \\ h_{21} & h_{22}\end{array}\right)=\left(\begin{array}{cc}\frac{3}{2} & -b \\ b & \frac{3}{2}\end{array}\right)$ : Choosing $T_{1}$ as above, the form of $H$ is kept, however with possibly nonvanishing $(2,1)$-entry and new $f_{i}, q_{i}$, namely

$$
\begin{array}{lll}
\bar{f}_{1}=f_{1}+b \tau_{3}-\frac{5}{2} \tau_{6} & \bar{q}_{1}=q_{1}+b \tau_{7}+\frac{7}{2} \tau_{8} \\
\bar{f}_{2}=f_{2}-\frac{5}{2} \tau_{3}-b \tau_{6} & \bar{q}_{2}=q_{2}+\frac{7}{2} \tau_{7}-b \tau_{8} .
\end{array}
$$

So the $f_{i}$ - and $q_{i}$-entries of $H$ can be made 0 , and also its $(2,1)$-entry by subtracting a multiple of $E$.

As to the translation parts we can achieve by TC-design that all $H_{i}=0$ and $E_{3}=$ $E_{4}=0$, arriving at $\|35\|$.

\section{Concluding remarks}

6.1. Similarity w.r.t. $\widetilde{\mathbf{S A}}$. So far, the similarity of subgroups of SA has been treated w.r.t. conjugation in GA. This has the advantage of a greater flexibility in the reduction process. If one prefers similarity of subgroups w.r.t. conjugation in $\widetilde{\mathbf{S A}}$, the results carry over without change, except in case $\|1\|$ where the homothetic images have to be added. The reason is the following:

If $\Gamma_{1}$ is a two-dimensional subgroup of $\mathbf{S A}$ with nondegenerate orbits then there is an element $\Gamma$ in the above list with $\Gamma_{1}=\mathbf{T} \Gamma \mathbf{T}^{-1}$ for some $\mathbf{T} \in \mathbf{G A}$. The $\mathbf{T}$ can be uniquely decomposed as $\mathbf{T}=\mathbf{T}_{0} \mathbf{P}_{r}$ where $\mathbf{P}_{r}:=(r I \| 0)_{1}$ is a linear homothety with $r>0$ and $\mathbf{T}_{0} \in \widehat{\mathbf{S A}}$. Then $\Gamma_{1}=\mathbf{T}_{0}\left(\mathbf{P}_{r} \Gamma \mathbf{P}_{r}^{-1}\right) \mathbf{T}_{0}^{-1}$. Thus, at most the groups $\mathbf{P}_{r} \Gamma \mathbf{P}_{r}^{-1}$ for $r>0$ have to be added to the resulting list of $\Gamma$ 's in order to obtain a list w.r.t. conjugation in $\widetilde{\mathbf{S A}}$.

However, in most cases $\mathbf{P}_{r} \Gamma \mathbf{P}_{r}^{-1}$ is $\widetilde{\mathbf{S A}}$-conjugate to $\Gamma$. For example, if the generators $\mathbf{L}$ of $\Gamma^{\bullet}$ have vanishing translation parts then $\mathbf{P}_{r} \mathbf{L} \mathbf{P}_{r}^{-1}=\mathbf{L}$, hence $\mathbf{P}_{r} \Gamma \mathbf{P}_{r}^{-1}=\Gamma$.

Otherwise, $\mathbf{P}_{r} \Gamma \mathbf{P}_{r}^{-1}$ is $\widetilde{\mathbf{S A}}$-conjugate to $\mathbf{P}_{s} \Gamma \mathbf{P}_{s}^{-1}(r, s>0)$ iff, for some $\mathbf{T}_{0} \in \widetilde{\mathbf{S A}}$, we have $\mathbf{P}_{r} \Gamma \mathbf{P}_{r}^{-1}=\mathbf{T}_{0} \mathbf{P}_{s} \Gamma \mathbf{P}_{s}^{-1} \mathbf{T}_{0}^{-1}$, i.e. $\mathbf{P}_{r}^{-1} \mathbf{T}_{0} \mathbf{P}_{s} \in \mathcal{F}_{\Gamma}$. Equivalently, there must exist a $\mathbf{T} \in \mathcal{F}_{\Gamma}$ with $|\operatorname{det} \mathbf{T}|=\frac{s^{4}}{r^{4}}$, i.e. the range $D(\Gamma)$ of $|\operatorname{det} \mathbf{T}|$ for $\mathbf{T} \in \mathcal{F}_{\Gamma}$ must contain $\frac{s^{4}}{r^{4}}$.

For all $\Gamma$ in the cases $\|2\|$ to $\|35\|$, one can check that $D(\Gamma)=\mathbf{R}^{+}$, so $\mathbf{P}_{r} \Gamma \mathbf{P}_{r}^{-1}$ is always $\widetilde{\mathbf{S A}}$-conjugate to $\Gamma$, and there is no change at all. For the $\Gamma$ of case $\|1\|$, one checks $D(\Gamma)=\{1\}$, so no two groups $\mathbf{P}_{r} \Gamma \mathbf{P}_{r}^{-1}$ and $\mathbf{P}_{s} \Gamma \mathbf{P}_{s}^{-1}$ are $\widetilde{\mathbf{S A}}$-conjugate, unless $r=s$. 
In any case, for a generator $\mathbf{L}=(L \| l)_{0}$ of $\Gamma^{\bullet}$, we have $\mathbf{P}_{r} \mathbf{L} \mathbf{P}_{r}^{-1}=(L \| r l)_{0}$, so there is only an effect on the translation parts. For an orbit $\gamma:=\Gamma x_{0}$ of $\Gamma$ with initial point $x_{0}$, the orbit $\gamma_{1}$ of $\Gamma_{1}:=\mathbf{P}_{r} \Gamma \mathbf{P}_{r}^{-1}$ with initial point $\mathbf{P}_{r} x_{0}$ is $\gamma_{1}=\Gamma_{1} \mathbf{P}_{r} x_{0}=\mathbf{P}_{r} \gamma$. Observe that, with $\gamma$, this $\gamma_{1}$ has again a regular premetric. Moreover, one sees that $\mathcal{F}_{\Gamma_{1}}=\mathbf{P}_{r} \mathcal{F}_{\Gamma} \mathbf{P}_{r}^{-1}$, and the same for the corresponding intersections with $\widetilde{\mathbf{S A}}$. This implies that the equivalence of two initial points $x_{0}, \widetilde{x}_{0}$ modulo $\mathcal{F}_{\Gamma} \cap \widetilde{\mathbf{S A}}$ is the same as the equivalence of the two initial points $\mathbf{P}_{r} x_{0}, \mathbf{P}_{r} \widetilde{x}_{0}$ modulo $\mathcal{F}_{\Gamma_{1}} \cap \widetilde{\mathbf{S A}}$. So the transition from $\Gamma$ to $\Gamma_{1}$ can be done by the following scheme: $(E \| e),(H \| h) \mapsto(E \| r e),(H \| r h)$ for the generators, $x_{0} \mapsto r x_{0}$ for the initial points, $\gamma \mapsto r \gamma$ for the orbits, the last two being expressed in $\mathbf{R}^{4}$. (As said above, this scheme has only to be applied in case $\|1\|$.)

6.2. Related result for hypersurfaces. Recently, M. Wermann [13], [14] classified the homogeneous hypersurfaces in the Blaschke-Berwald geometry of $\mathbf{R}^{4}$, generated by threedimensional subgroups of $\mathbf{S A}(4, \mathbf{R})$.

\section{References}

[1] C. Burstin und W. Mayer, Die Geometrie zweifach ausgedehnter Mannigfaltigkeiten $F_{2}$ im affinen Raum $R_{4}$, Math. Z. 27 (1927), 373-407.

[2] A. A. Kirillov, Elements of the Theory of Representations, Springer, 1976 (English translation of the Russian original, 1954).

[3] H. Liu, Indefinite equi-centroaffinely homogeneous surfaces with vanishing Pick-invariant in $\mathbf{R}^{4}$, Hokkaido Math. J. 26 (1997), 225-251.

[4] K. Nomizu and L. Vrancken, A new equiaffine theory for surfaces in $\mathbf{R}^{4}$, Intern. J. Math. 4 (1993), 127-165.

[5] R. Walter, Homogeneity for surfaces in four-dimensional vector space geometry, Geom. Dedicata 71 (1998), 129-178.

[6] R. Walter, Homogeneous parabolic surfaces in $\mathbf{R}^{4}$, Contr. Alg. Geom. 41 (2000), 159-180.

[7] R. Walter, Gauge theory, isotropy, and surfaces in affine 4-space, in preparation.

[8] C. P. Wang, A unified equiaffine theory for surfaces in $\mathbf{R}^{4}$. I: The definite surfaces, in: Geometry and Topology of Submanifolds, VI, World Scientific, 1994, 210-223.

[9] C. P. Wang, Equiaffine theory of surfaces in $\mathbf{R}^{4}$ Dissertation TU Berlin, 1995.

[10] C. P. Wang, A unified equiaffine theory for surfaces in $\mathbf{R}^{4}$. II: Indefinite surfaces, Results Math. 27 (1995), 198-205.

[11] C. P. Wang, Homogeneous submanifolds, in: Geometry and Topology of Submanifolds, VIII, World Scientific, 1996.

[12] C. P. Wang, The classification of equiaffine indefinite flat homogeneous surfaces in $\mathbf{R}^{4}$, Geom. Dedicata 65 (1997), 323-353.

[13] M. Wermann, Homogene Hyperflächen im vierdimensionalen äqui-affinen Raum mit kommutativer Gruppe, Diplomarbeit Univ. Dortmund, 1998.

[14] M. Wermann, Homogene Hyperflächen im vierdimensionalen äqui-affinen Raum, thesis preprint, 1-219. 